Document downloaded from:

http://hdl.handle.net/10251/78196

This paper must be cited as:

Martínez Casas, J.; Mazzola, L.; Baeza González, LM.; Bruni, S. (2013). Numerical Estimation of Stresses in Railway Axles Using a Train-Track Interaction Model. International Journal of Fatigue. 47:18-30. doi:10.1016/j.ijfatigue.2012.07.006.

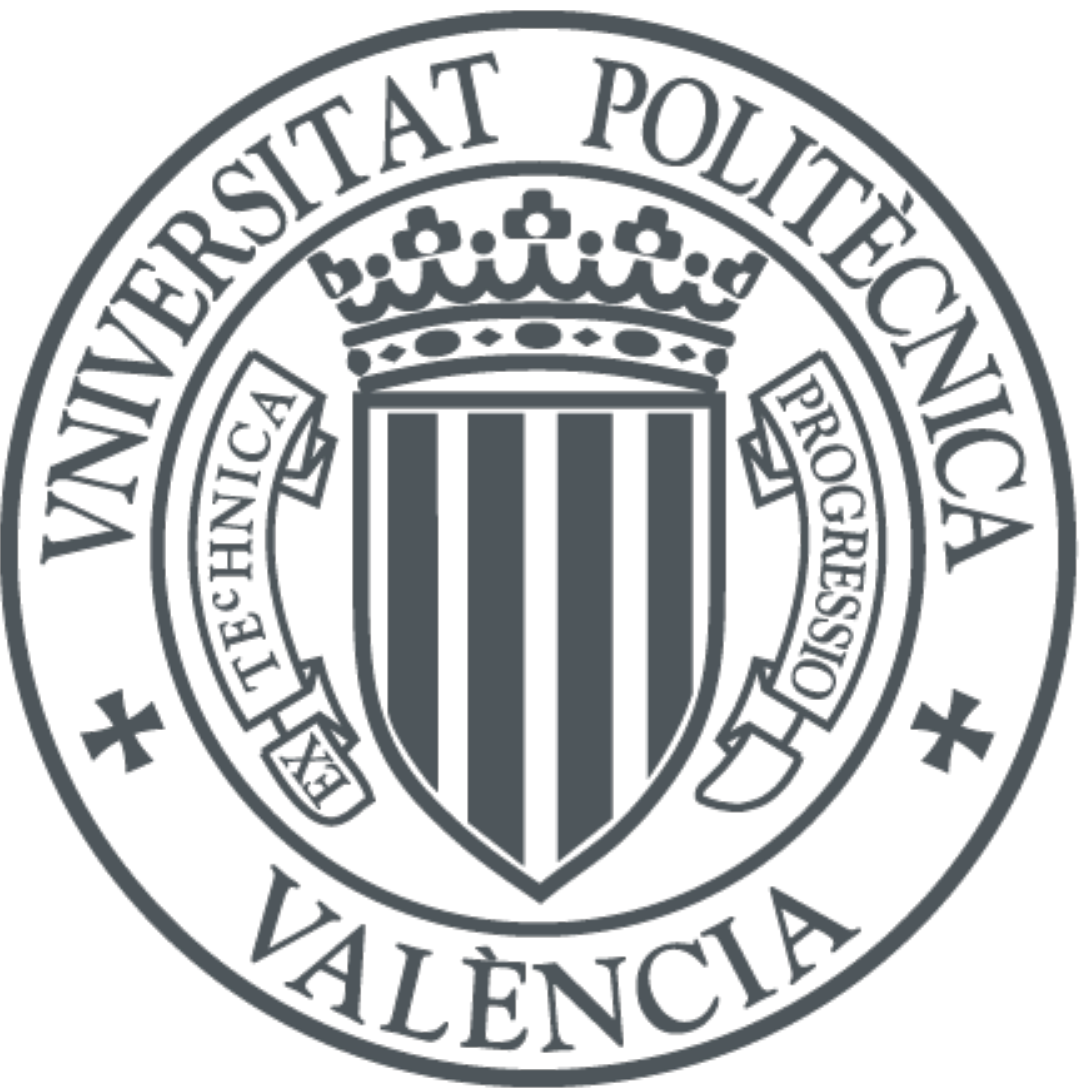

The final publication is available at

http://dx.doi.org/10.1016/j.ijfatigue.2012.07.006

Copyright Elsevier

Additional Information 


\title{
Numerical Estimation of Stresses in Railway Axles Using a Train-
}

\section{Track Interaction Model}

\author{
Authors: José Martínez-Casas ${ }^{1}$, Laura Mazzola ${ }^{2}$, Luis Baeza ${ }^{1}$, Stefano Bruni $^{2}$ \\ Affiliation: \\ ${ }^{1}$ Centro de Investigación en Tecnología de Vehículos, Universidad Politécnica de Valencia, Camino \\ de Vera s/n, 46022 Valencia - Spain \\ ${ }^{2}$ Dipartimento di Meccanica, Politecnico di Milano, Via La Masa 1, 20156 Milano, Italy \\ E-mail corresponding author: stefano.bruni@mecc.polimi.it
}

\begin{abstract}
The fatigue design of railway axles requires that the stresses arising in the axle in real service are accurately quantified. This paper describes a method to compute the dynamic stresses arising in railway axles as the effect of train-track interaction, based on the numerical simulation of the dynamic interaction between a flexible wheelset and a flexible track. The wheelset is modelled as a flexible rotating body using an Eulerian approach, whereas track is regarded as an infinite periodic system with the rail modelled as a Timoshenko beam resting on discrete elastic supports, considering the inertia associated with the sleepers.

The paper presents an application of the proposed procedure to the calculation of the dynamic stresses caused in the axle by different types of geometric imperfection occurring on the wheel and rail surfaces, considering the cases of a single harmonic rail corrugation, random rail roughness and a wheelflat.
\end{abstract}

\section{Keywords}

Railway vehicles, wheelset design, wheelset axle fatigue, dynamic loads, train-track interaction, rail roughness, wheelflat 


\section{Introduction}

Railway axles durability is a key issue in designing and correctly maintaining railway vehicles, to ensure that the highest safety standards are met and, at the same time, to optimise life-cycle costs from a system point of view, i.e. considering not only the vehicle but also the interacting infrastructure. From the single point of view of fatigue resistance, the axle design should tend towards increasing the size to reduce stresses, but this component also represents a significant contribution to the wheelset un-sprung mass, which, on the contrary, shall be minimised to reduce the generation of dynamic force at wheel-rail contact and hence damage in the track and in the wheels, especially for high-speed trains.

At present, wheelset axles are designed for infinite fatigue life, however a small number of axle failures due to fatigue continues to be reported, sometimes with catastrophic consequences. One of the reasons which have been proposed to explain these unforeseen failures is that the loads assumed by the standard for the purpose of fatigue design verification do not fully reflect the actual service loads of the vehicle, which are largely depending on the vehicle design parameters (e.g. unsprung masses, suspension stiffness and damping, ...) and on the vehicle service profile $[1,2]$. The precise knowledge of service loads is also pivotal to the definition of appropriate intervals for the nondestructive inspection of railway axles [3, 4], which are nowadays part of standard maintenance practice to remove from service cracked axles before failure.

Detailed information on the service loads is often derived experimentally, using instrumented wheelsets [5 - 7]. This approach however presents some drawbacks: first of all, the physical measure is not applicable at the design stage of a new vehicle, setting-up and running the tests is expensive and demanding due to the harsh measuring environment and extensive test campaigns are required to cover all service conditions (different lines and speeds, operation at tare / full load, new / worn wheel profiles, ...). Furthermore, the experimental measure of the service load is normally 
not able to fully explain the causes of extreme loads, which result in stress peaks mostly affecting the fatigue resistance of the axle and crack propagation.

For this reason, in the last year attempts have been made to define procedures based on the use of multi-body models of a railway vehicle or train set, to provide a numerical estimate of the service loads [6 - 8]. This approach can be used to complement measurements, e.g. addressing service conditions not covered by the tests and to provide a better physical understanding of the factors influencing the service spectra, including (among others) vehicle design parameters, the condition of the wheel and rail surfaces and the service scenario. To the Authors' knowledge, these approaches have however until now been developed considering the wheelset as a rigid body. Hence, axle stresses can be correctly computed only in the low-frequency range (approximately 0 $20 \mathrm{~Hz}$ ), whereas their high-frequency components, mainly responsible for the occurrence of stress peaks, are excluded from the analysis [8].

This paper aims therefore at establishing a method to define numerically the stresses in a railway axle, including the high frequency components, as function of vehicle-track parameters and of the vehicle's running condition, thereby extending the scope and detail of the existing numerical approaches to predict the wheelset service loads. To this end, a model of a flexible wheelset interacting with a flexible track in tangent track is derived and used to simulate typical service scenarios for a high-speed railway vehicle. The wheelset is modelled as a flexible rotating body, using an Eulerian approach to take advantage from axial symmetry and modal synthesis to reduce the size of the problem. The track is modelled as an infinite periodic system, with the rail modelled as a Timoshenko beam resting on discrete elastic supports, considering the inertia associated with the sleepers. The numerical simulation considers the effect of geometric irregularities appearing on the rail and wheel surfaces, allowing to consider the effect of e.g. rail corrugation, rail dips, wheel polygonalisation, wheel flats etc. 
Train-track interaction models including a detailed description of the wheelset as an elastically deformable body have been proposed by several authors. Some models, e.g. [9] are defined in the frequency domain and derive the dynamic forces at wheel-rail contact by combining the wheelset and track frequency response functions with the assumed rail corrugation. This approach is inherently linear and does not allow to consider the rails as discretely supported. Modelling approaches which incorporate a discrete-support track model and the effect of nonlinearities in the track and in wheel-rail contact have been developed in Refs. [10-14]: in this case, the train-track model is defined in the time domain and the wheelset and track models are defined using the finite element method, introducing a reduction of the degrees of freedom using mode superposition.

These approaches have been mostly applied to the study of damage phenomena in the track and at wheel-rail interface, and therefore focus on the definition of wheel-rail contact forces rather than axle stresses. Therefore, a simplified model of the wheelset axle (sometimes based on beam elements) can be used. Furthermore, the effect of wheelset rotation is neglected or considered with some approximation, whereas this effect may importantly affect the wheelset resonance conditions, which in turn can be a cause of extreme stress peaks arising in the axle: the present paper aims therefore at developing a modelling and simulation approach specifically tailored on the prediction of axle stresses

The paper describes the wheelset-track interaction model developed and the procedure to compute the dynamic stresses in the axle. Results of the numerical procedure are presented for different excitation cases including rail corrugation having different wavelength and a wheelflat, and considering the effect of wheelset speed. 


\section{THE VEHICLE-TRACK INTERACTION MODEL}

The vehicle - track interaction model, see Figure 1, is defined adopting a sub-structuring technique $[15,16]$, according to which the whole system is divided into substructures: the vehicle, the rails and the rail supports. For each substructure, the equations of motion are written separately, and interaction effects between vehicle and track are represented by the wheel-rail contact forces and by the forces generated at the railpads [15]. The simulation approach is developed considering the motion of the wheelset in tangent track.

Given the range of frequencies addressed, the vehicle model is confined to one wheelset with primary suspension, see section 2.1. The vibration of the flexible wheelset is expressed using Eulerian modal coordinates, see Section 3, taking advantage form the axial symmetry of the body. Furthermore the modal approach is chosen to describe the wheelset motion in order to reduce the computational effort required by the simulation.

The track is modelled by means of a cyclic approach, which provides some benefit with respect to classical track modelling or the finite one. The sub-structuring technique is still adopted here to simplify the system modelling; a detailed description of the track is provided in section 2.2.

\subsection{The Vehicle Model}

The dynamic stresses arising in the wheelset axle are mostly related with train-track interaction effects in the frequency range above $20 \mathrm{~Hz}$, which are excited by short wavelength geometric imperfections in the wheel and rail profiles and by singularities such as rail dips and wheel flats. In this frequency range the dynamics of the sprung masses (bogie frame and car body) are effectively isolated from the motion of the un-sprung masses (wheelsets and axle boxes) on account of the mechanical filter introduced by the suspensions. Therefore, the vehicle model used in this paper considers one single wheelset, modelled as an elastically flexible body, and the primary 
suspension, represented using visco-elastic lumped parameter elements. The static load associated with the gravitational forces acting on the bogie and on the carbody masses is represented by two static forces applied through the primary suspension on the two sides of the wheelset.

\subsection{The Track Model}

The track is modelled by means of a cyclic approach, where a finite section of the track is defined, introducing cyclic boundary conditions at the ends of the model, hence the track, see Figure 2, can be interpreted as an infinite track, negotiated by an infinite set of identical vehicles, uniformly distributed in such a way that each vehicle is set at a constant distance L apart from the adjacent ones. Due to the periodicity of the structure and of the loading conditions, the study is reduced to a single section having finite length $\mathrm{L}$, whose value is set large enough to avoid interaction between the vehicles.

The model adopted for the different track components is illustrated in Figure 3: the rails are modelled as Timoshenko beams, including bending deformations in vertical/lateral directions, as well as torsional deformations. Rail vibration is introduced in terms of modal superposition for the unconstrained rail with cyclic boundary conditions, hence resulting into a set of de-coupled 1-d.o.f. equations. Moreover due to the symmetry of the sub-system with respect to the track centreline, only one rail is modelled in the present study.

The discrete rail supports are introduced in the form of lumped parameter systems, see Figure 3 right. The rail pads are modelled as lumped visco-elastic elements generating the interaction forces between the rails and the sleepers, represented as lumped masses. Ballast dynamics is neglected here, being not relevant for the stress analysis on the wheelset, but the equivalent ballast stiffness and damping are accounted for by means of lumped spring and dashpot elements. 


\subsection{The Model of Wheel-Rail Contact Forces}

The track model is coupled with the flexible wheelset via the wheel-rail contact forces, which are expressed as function of the relative wheel-rail displacement and velocity in the contact patch. In the numerical model presented here, the theory of Hertz is adopted to define the normal contact force component and FASTSIM [17] is used to define the tangential contact forces as function of the normal contact force and of the creepage components.

At each time step, the displacement and velocity of the wheel in the contact point are obtained using Eq. (1) evaluated at the contact point position.

\section{THE FLEXIBLE WHEELSET MODEL}

In order to model the kinematics of the flexible wheelset, two configurations (undeformed and deformed) are defined. The undeformed configuration is associated with the spinning velocity of the wheelset (at constant angular velocity $\Omega$ ). The deformed configuration considers the flexibility and small rigid solid displacements. The displacement field relates the deformed configuration with the undeformed configuration as it will be shown in Eq. (1).

The coordinates that are implemented in the wheelset model do not follow the material points of the solid which is the commonest procedure in Mechanics, nevertheless they are associated with spatial points (Eulerian approach). Let $\mathbf{u}$ an Eulerian vector coordinate in a fixed coordinate frame. Any property of the solid $\varphi(\mathbf{u}, t)$ corresponds to the material point of the solid whose undeformed configuration is in the spatial point $\mathbf{u}$ at instant $t$. Following this criterion, the displacement field is defined by means of the following formula:

$$
\mathbf{r}=\mathbf{u}+\mathbf{w}(\mathbf{u}, t),
$$

where $\mathbf{r}$ is the final position of the particle, and $\mathbf{w}$ is the displacements associated with flexibility and small rigid body displacements. 
The coordinate frame is chosen so that the spin rotation is in the second axle. The following matrices are defined as follows:

$$
\mathbf{J}=\left(\begin{array}{ccc}
0 & 0 & 1 \\
0 & 0 & 0 \\
-1 & 0 & 0
\end{array}\right) ; \quad \mathbf{E}=\left(\begin{array}{ccc}
1 & 0 & 0 \\
0 & 0 & 0 \\
0 & 0 & 1
\end{array}\right)
$$

The angular velocity tensor verifies:

$$
\tilde{\boldsymbol{\Omega}}=\left(\begin{array}{ccc}
0 & 0 & \Omega \\
0 & 0 & 0 \\
-\Omega & 0 & 0
\end{array}\right)=\Omega \mathbf{J} ; \text { and } \tilde{\boldsymbol{\Omega}} \tilde{\boldsymbol{\Omega}}=-\Omega^{2} \mathbf{E}
$$

The velocity due to the rigid body spinning is:

$$
\mathbf{v}=\left(\begin{array}{lll}
v_{1} & v_{2} & v_{3}
\end{array}\right)^{\mathrm{T}}=\tilde{\mathbf{\Omega}} \mathbf{u}=\Omega \mathbf{J} \mathbf{u}=\Omega \tilde{\mathbf{u}},
$$

where $\tilde{\mathbf{u}}=\left(\begin{array}{lll}u_{3} & 0 & -u_{1}\end{array}\right)^{\mathrm{T}}$. The velocity of the particle is computed through the material derivative of $\mathbf{r}$, and that is

$$
\frac{\mathrm{D} \mathbf{r}}{\mathrm{D} t}=\frac{\mathrm{D} \mathbf{u}}{\mathrm{D} t}+\frac{\mathrm{D} \mathbf{w}}{\mathrm{D} t}=\sum_{i} v_{i} \frac{\partial \mathbf{u}}{\partial u_{i}}+\dot{\mathbf{w}}+\sum_{i} v_{i} \frac{\partial \mathbf{w}}{\partial u_{i}}=\Omega \mathbf{J} \mathbf{u}+\dot{\mathbf{w}}+\Omega \sum_{i} \tilde{u}_{i} \frac{\partial \mathbf{w}}{\partial u_{i}}
$$

In order to calculate the kinetic energy of the solid, the square of the particle velocity Eq. (5) has to be obtained. It has the following formula:

$$
\begin{aligned}
\frac{\mathrm{Dr}}{\mathrm{D} t} \frac{\mathrm{D} \mathbf{r}}{\mathrm{D} t} & =\Omega^{2} \mathbf{u}^{\mathrm{T}} \mathbf{E} \mathbf{u}+\dot{\mathbf{w}}^{\mathrm{T}} \dot{\mathbf{w}}+\Omega^{2}\left(\sum_{i} \tilde{u}_{i} \frac{\partial \mathbf{w}^{\mathrm{T}}}{\partial u_{i}}\right)\left(\sum_{i} \tilde{u}_{i} \frac{\partial \mathbf{w}}{\partial u_{i}}\right) \\
& +2 \Omega \dot{\mathbf{w}}^{\mathrm{T}} \mathbf{J} \mathbf{u}+2 \Omega^{2}\left(\sum_{i} \tilde{u}_{i} \frac{\partial \mathbf{w}^{\mathrm{T}}}{\partial u_{i}}\right) \mathbf{J} \mathbf{u}+2 \Omega \dot{\mathbf{w}}^{\mathrm{T}}\left(\sum_{i} \tilde{u}_{i} \frac{\partial \mathbf{w}}{\partial u_{i}}\right) .
\end{aligned}
$$

The next modal approach is adopted:

$$
\mathbf{w}(\mathbf{u}, t)=\mathbf{\Phi}(\mathbf{u}) \mathbf{q}(t),
$$

being $\boldsymbol{\Phi}(\mathbf{u})$ the mode shape functions matrix of the free-boundary wheelset. The small rigid displacements of the solid are considered in this approach through the rigid modes of the wheelset. It must be pointed out that the mode shape functions do not depend on time since the rotation of the solid does not change the mode shapes functions in fixed coordinates, because the axial symmetry 
of the wheelset. The use of the mode shapes in fixed coordinates as basis function when the solid is

in motion is called Eulerian modal approach. Once the formula Eq. (7) is applied in Eq.(6), the kinetic energy results in the following expression:

$$
\begin{aligned}
E_{k} & =\frac{1}{2} \int_{V o l} \rho \frac{\mathrm{Dr}}{\mathrm{D} t} \frac{\mathrm{D} \mathbf{r}}{\mathrm{D} t} \mathrm{~d} v=\frac{1}{2} \dot{\mathbf{q}}^{\mathrm{T}} \int_{V o l} \rho \boldsymbol{\Phi}^{\mathrm{T}} \mathbf{\Phi} \mathrm{d} v \dot{\mathbf{q}}+\frac{1}{2} \Omega^{2} \int_{V o l} \rho \mathbf{u}^{\mathrm{T}} \mathbf{E} \mathbf{u} \mathrm{d} v \\
& +\frac{1}{2} \Omega^{2} \mathbf{q}^{\mathrm{T}} \int_{V o l} \rho\left(\sum_{i} \tilde{u}_{i} \frac{\partial \boldsymbol{\Phi}^{\mathrm{T}}}{\partial u_{i}}\right)\left(\sum_{i} \tilde{u}_{i} \frac{\partial \boldsymbol{\Phi}}{\partial u_{i}}\right) \mathrm{d} v \mathbf{q}+\Omega \dot{\mathbf{q}}^{\mathrm{T}} \int_{V o l} \rho \boldsymbol{\Phi}^{\mathrm{T}} \mathbf{J} \mathbf{u} \mathrm{d} v \\
& +\Omega^{2} \mathbf{q}^{\mathrm{T}} \int_{V o l} \rho\left(\sum_{i} \tilde{u}_{i} \frac{\partial \boldsymbol{\Phi}^{\mathrm{T}}}{\partial u_{i}}\right) \mathbf{J} \mathbf{u} \mathrm{d} v+\Omega \dot{\mathbf{q}}^{\mathrm{T}} \int_{V o l} \rho \boldsymbol{\Phi}^{\mathrm{T}}\left(\sum_{i} \tilde{u}_{i} \frac{\partial \boldsymbol{\Phi}}{\partial u_{i}}\right) \mathrm{d} v \mathbf{q} .
\end{aligned}
$$

Two terms of Lagrange's equation are computed as follows:

$$
\begin{aligned}
\frac{\mathrm{D}}{\mathrm{D} t}\left(\frac{\partial E_{k}}{\partial \dot{\mathbf{q}}}\right)^{\mathrm{T}}= & \int_{V o l} \rho \boldsymbol{\Phi}^{\mathrm{T}} \boldsymbol{\Phi} \mathrm{d} v \ddot{\mathbf{q}}+\Omega \int_{V o l} \rho \boldsymbol{\Phi}^{\mathrm{T}}\left(\sum_{i} \tilde{u}_{i} \frac{\partial \boldsymbol{\Phi}}{\partial u_{i}}\right) \mathrm{d} v \dot{\mathbf{q}}-\Omega^{2} \int_{V o l} \rho \boldsymbol{\Phi}^{\mathrm{T}} \mathbf{E} \mathbf{u} \mathrm{d} v \\
& +\Omega \int_{V o l} \rho\left(\sum_{i} \tilde{u}_{i} \frac{\partial \boldsymbol{\Phi}^{\mathrm{T}}}{\partial u_{i}}\right) \mathbf{\Phi} \mathrm{d} v \dot{\mathbf{q}}+\Omega \int_{V o l} \rho \boldsymbol{\Phi}^{\mathrm{T}}\left(\sum_{i} \tilde{u}_{i} \frac{\partial \boldsymbol{\Phi}}{\partial u_{i}}\right) \mathrm{d} v \dot{\mathbf{q}} \\
& +\Omega^{2} \int_{V o l} \rho\left(\sum_{i} \tilde{u}_{i} \frac{\partial \boldsymbol{\Phi}^{\mathrm{T}}}{\partial u_{i}}\right) \mathbf{J} \mathbf{u} \mathrm{d} v-\Omega^{2} \int_{V o l} \rho \boldsymbol{\Phi}^{\mathrm{T}}\left(\sum_{i=1,3} u_{i} \frac{\partial \boldsymbol{\Phi}}{\partial u_{i}}\right) \mathrm{d} v \mathbf{q} \\
& +\Omega^{2} \int_{V o l} \rho\left(\sum_{i} \tilde{u}_{i} \frac{\partial \boldsymbol{\Phi}^{\mathrm{T}}}{\partial u_{i}}\right)\left(\sum_{i} \tilde{u}_{i} \frac{\partial \boldsymbol{\Phi}}{\partial u_{i}}\right) \mathrm{d} v \mathbf{q}+\Omega^{2} \int_{V o l} \rho \boldsymbol{\Phi}^{\mathrm{T}}\left(\sum_{j} \sum_{i} \tilde{u}_{i} \tilde{u}_{j} \frac{\partial^{2} \boldsymbol{\Phi}}{\partial u_{i} \partial u_{j}}\right) \mathrm{d} v \mathbf{q} \\
\left(\frac{\partial E_{k}}{\partial \mathbf{q}}\right)^{\mathrm{T}} & =\Omega^{2} \int_{V o l} \rho\left(\sum_{i} \tilde{u}_{i} \frac{\partial \boldsymbol{\Phi}^{\mathrm{T}}}{\partial u_{i}}\right)\left(\sum_{i} \tilde{u}_{i} \frac{\partial \boldsymbol{\Phi}}{\partial u_{i}}\right) \mathrm{d} v \mathbf{q} \\
& +\Omega^{2} \int_{V o l} \rho\left(\sum_{i} \tilde{u}_{i} \frac{\partial \boldsymbol{\Phi}^{\mathrm{T}}}{\partial u_{i}}\right) \mathbf{J} \mathbf{u} \mathrm{d} v+\Omega \int_{V o l} \rho\left(\sum_{i} \tilde{u}_{i} \frac{\partial \boldsymbol{\Phi}^{\mathrm{T}}}{\partial u_{i}}\right) \boldsymbol{\Phi} \mathrm{d} v \dot{\mathbf{q}}
\end{aligned}
$$

Finally, the equation of motion is obtained:

$$
\begin{aligned}
\frac{\mathrm{D}}{\mathrm{D} t}\left(\frac{\partial E_{k}}{\partial \dot{\mathbf{q}}}\right)^{\mathrm{T}}-\left(\frac{\partial E_{k}}{\partial \mathbf{q}}\right)^{\mathrm{T}} & =\ddot{\mathbf{q}}+2 \Omega \int_{V o l} \rho \boldsymbol{\Phi}^{\mathrm{T}}\left(\sum_{i} \tilde{u}_{i} \frac{\partial \boldsymbol{\Phi}}{\partial u_{i}}\right) \mathrm{d} v \dot{\mathbf{q}}+\Omega^{2} \int_{V o l} \rho \boldsymbol{\Phi}^{\mathrm{T}}\left(\sum_{j} \sum_{i} \tilde{u}_{i} \tilde{u}_{j} \frac{\partial^{2} \mathbf{\Phi}}{\partial u_{i} \partial u_{j}}\right) \mathrm{d} v \mathbf{q} \\
& -\Omega^{2} \int_{V o l} \rho \boldsymbol{\Phi}^{\mathrm{T}}\left(\sum_{i=1,3} u_{i} \frac{\partial \boldsymbol{\Phi}}{\partial u_{i}}\right) \mathrm{d} v \mathbf{q}-\Omega^{2} \int_{V o l} \rho \boldsymbol{\Phi}^{\mathrm{T}} \mathbf{E} \mathbf{u} \mathrm{d} v .
\end{aligned}
$$

The modal properties are computed from a finite element (FE) model, therefore it is convenient to use the FE methodology for computing the equation of motion numerically. The mode shape functions are obtained into the $e$-th element of the FE mesh as follows 


$$
\boldsymbol{\Phi}(\mathbf{u})=\mathbf{N}^{e}(\mathbf{u}) \boldsymbol{\Phi}_{F E}^{e},
$$

being $\mathbf{N}^{e}(\mathbf{u})$ the basis function matrix of the $e$-th element, and $\boldsymbol{\Phi}_{F E}^{e}$ the mode shapes computed in the nodes of the $e$-th element through the FE model.

One first step in the FE method is to compute each integral on the volume of the solid as the sum of integrals on the volume of the FE elements, that is:

$$
\int_{V o l}^{\bullet} \mathrm{d} v=\sum_{\text {elements }} \int_{V o l^{e}} \bullet \mathrm{d} v
$$

This approach can be performed if the integrand is bounded in the element edges. Once this step is complete, the method in centred on obtaining the matrices of the elements. These matrices have to be assembled in global matrices, following the standard FE assembling technique. The first matrix of the element in Eq. (11) is obtained by means of the approach in Eq. (12) as follows:

$$
2 \Omega \int_{V o l^{e}} \rho \boldsymbol{\Phi}^{\mathrm{T}}\left(\sum_{i} \tilde{u}_{i} \frac{\partial \boldsymbol{\Phi}}{\partial u_{i}}\right) \mathrm{d} v=2 \Omega \boldsymbol{\Phi}_{F E}^{e \mathrm{~T}} \int_{V o l^{e}} \rho \mathbf{N}^{e^{\mathrm{T}}}\left(\sum_{i} \tilde{u}_{i} \frac{\partial \mathbf{N}^{e}}{\partial u_{i}}\right) \mathrm{d} v \boldsymbol{\Phi}_{F E}^{e},
$$

being defined $\mathbf{V}^{e}$ as the corresponding matrix of the element, that is:

$$
\mathbf{V}^{e}=\int_{V o l^{e}} \rho \mathbf{N}^{e^{\mathrm{T}}}\left(\sum_{i} \tilde{u}_{i} \frac{\partial \mathbf{N}^{e}}{\partial u_{i}}\right) \mathrm{d} v
$$

Following the same procedure than Eq. (14), the remaining matrices of the elements are obtained :

$$
\begin{gathered}
\mathbf{A}^{e}=\int_{V o l^{e}} \rho \mathbf{N}^{e^{\mathrm{T}}}\left(\sum_{j} \sum_{i} \tilde{u}_{i} \tilde{u}_{j} \frac{\partial^{2} \mathbf{N}^{e}}{\partial u_{i} \partial u_{j}}\right) \mathrm{d} v, \\
\mathbf{C}^{e}=\int_{V o l^{e}} \rho \mathbf{N}^{e^{\mathrm{T}}}\left(\sum_{i=1,3} u_{i} \frac{\partial \mathbf{N}^{e}}{\partial u_{i}}\right) \mathrm{d} v, \\
\mathbf{c}^{e}=\int_{V o l^{e}} \rho \mathbf{N}^{e^{\mathrm{T}}} \mathbf{E} \mathbf{u} \mathrm{d} v .
\end{gathered}
$$

The term where $\mathbf{V}^{e}$ appears can be identified as inertial force due to Coriolis acceleration associated with the convective velocity. Matrix $\mathbf{A}^{e}$ is related to convective acceleration. Matrix $\mathbf{C}^{e}$ is associated with centrifugal forces that appear after deformation of the solid. Finally, column 
The presence of the second derivative in Eq. (16) does not guarantee the convergence to the integral (because there is $\mathrm{C}^{0}$ continuity between FE elements). However, the convergence can be proved if one integrates Eq. (16) by parts.

The terms of Lagrange's equation related with non - conservative forces and elastic energy are computed like standard modal approach procedure (because Eulerian displacements and virtual displacements do not depend on time). The matrices of the elements (e.g. $\mathbf{A}^{e}$ ) are assembled into the global matrices (giving matrix $\mathbf{A}$ ), and the following equation of motion is found

$$
\ddot{\mathbf{q}}+2 \Omega \boldsymbol{\Phi}_{F E}^{\mathrm{T}} \mathbf{V} \boldsymbol{\Phi}_{F E} \dot{\mathbf{q}}+\left(\mathbf{D}+\Omega^{2} \boldsymbol{\Phi}_{F E}^{\mathrm{T}}(\mathbf{A}-\mathbf{C}) \boldsymbol{\Phi}_{F E}\right) \mathbf{q}=\Omega^{2} \boldsymbol{\Phi}_{F E}^{\mathrm{T}} \mathbf{c}+\mathbf{Q},
$$

being $\mathbf{Q}$ the generalized forces, and $\mathbf{D}$ a diagonal matrix that contains the square of the undamped natural frequencies of the free-boundary solid.

\subsection{Calculation of Stresses in the Wheelset}

The simulation of train-track interaction is finalized in this paper towards the calculation of dynamic stresses arising in the wheelset axle. To perform the stress calculation, first of all some sections of interest are identified along the axle. These sections were chosen sufficiently far from diameter changes, to avoid stress concentration effects which cannot be captured with the simple mesh adopted, see Figure 4. In this paper, results are reported for one single section of the axle, which is set at mid distance between the central brake disk and one of the side brake disks, see Figure 5 .

In order to derive the dynamic stresses, the simulation results in terms of the Eulerian modal coordinates vector $\mathrm{q}(\mathrm{t})$ are processed according to Eq. (1) to derive the strains and stresses in different spatial points along the exterior surface of the considered axle section. Then, one material 
point is chosen in the section and followed during the rotation of the wheelset, to derive the time history of the stress in the selected point.

\section{RESULTS}

In this section, results of train-track interaction calculations are shown considering different sources of excitation: a single harmonic rail corrugation, random rail roughness and a wheelflat. The case study considered here refers to the trailed car of a concentrated power train for high-speed passenger service. The vehicle is equipped with a solid axle wheelset with monobloc, light design wheels. The track considered features UIC60 rails and track parameters are based on the EUROBALT project, considering a "stiff” track.

Table 1 summarises the input data used to set-up the simulation model. In all analyses reported below (unless those considering the wheelset as a rigid body), the first 100 modes of vibration of the flexible wheelset are considered, covering the frequency range up to $2.7 \mathrm{kHz}$ approximately.

\section{Results are shown starting from the simple excitation case represented by a single harmonic rail} corrugation and then extended to the cases of random rail corrugation and wheelflat.

\subsection{Results for single harmonic rail corrugation}

The first excitation case considered is rail corrugation having sinusoidal waveform. Different corrugation wavelengths are considered, including values exciting some resonances of the track and wheelset. The first case considered refers to a corrugation wavelength of $60 \mathrm{~mm}$ (i.e. one tenth of the sleeper bay). In Figure 6 the vertical contact force generated by the wheelset travelling on the corrugated rail is plotted as function of the distance travelled for different vehicle speeds (100 and $300 \mathrm{~km} / \mathrm{h}$ ) and for different corrugation amplitudes. In the upper subfigure, a corrugation peak-topeak depth of $4.84 \mu \mathrm{m}$ is considered, corresponding to the limit amplitude defined by the ISO 3095 
standard. For the higher speed, the result obtained for the case of a rigid wheelset (i.e. excluding from the analysis all modes except the rigid ones) is also shown, to evaluate the effect of wheelset flexibility. Finally, the rail roughness profile is also reported in the figure using an appropriate scaling and offset to obtain a proper visualisation.

Two harmonic contents are observed in the vertical contact force, the largest one having the same wavelength as the rail corrugation and a second one with wavelength equal to the sleeper bay. This second harmonic component is due to the periodic variation of the rail stiffness seen by the wheelset as the consequence of the discrete rail support. The amplitude of the dynamic contact force component having the same wavelength as the corrugation is highly influenced by the wheelset speed, being approximately three times greater at $300 \mathrm{~km} / \mathrm{h}$ than at $100 \mathrm{~km} / \mathrm{h}$. The contact force is also highly affected by wheelset flexibility: the comparison of the results obtained for the rigid and flexible wheelset at the speed of $300 \mathrm{~km} / \mathrm{h}$ shows that neglecting wheelset flexibility leads to overestimating the peak-to-peak amplitude of the dynamic force by $25 \%$ approximately. This is due to the fact that at the considered excitation frequency of $1390 \mathrm{~Hz}$ approximately (corresponding to the ratio of the wheelset speed over the corrugation wavelength) the wheelset mass participating to the vertical motion is lower than the whole mass of the wheelset due to flexibility effects, a mechanism which is not captured by the rigid wheelset model.

The corrugation peak-peak amplitude of $4.84 \mu \mathrm{m}$ is relatively small compared to the typical amplitude of rail corrugation, which is in the range of tens or even hundreds of $\mu \mathrm{m}$. Indeed, ISO 3095 limit is defined for a random corrugated profile including several wavelengths which altogether would lead to a greater peak-peak amplitude. Therefore, in the lower subfigure of Figure 6 the vertical contact force obtained for the flexible wheelset at $300 \mathrm{~km} / \mathrm{h}$ is compared for two corrugation amplitudes, one corresponding to the ISO 3095 limit (same as in the upper subfigure) and the other ten times greater; this comparison also allows to assess the importance of non-linear 
effects in the case considered. For the larger corrugation amplitude, the peak-to peak amplitude of the vertical force is approximately $93 \mathrm{kN}$, and leads to a maximum wheel overloading / unloading which is approximately $78 \%$ of the static load. For the case considered here, non-linear effects appear to play a relatively marginal role, since the maximum dynamic variation of the vertical load for the larger corrugation amplitude is with good approximation ten times greater than for the small corrugation amplitude. However, the force fluctuation component having the same wavelength as the sleeper bay is in this case less visible (note that the amplitude of excitation associated with this effect does not change with the corrugation amplitude).

Figure 7 shows the y-axis normal stress in the studied section of the axle (cfr. Fig. 5), plotted as function of the wheelset rotation for the same cases considered in Figure 6. On the left, the effect of vehicle speed is analysed by comparing the results obtained at 100 and $300 \mathrm{~km} / \mathrm{h}$ for the same corrugation amplitude corresponding to the ISO 3095 limit: the waveform of the stress is with good approximation sinusoidal with $2 \pi$ periodicity, and the peak-peak amplitude is increased from 78.3 MPa to $88 \mathrm{MPa}$ when the speed is increased from 100 to $300 \mathrm{~km} / \mathrm{h}$. On the right, the effect of corrugation amplitude is considered by comparing the two results obtained for the two corrugation amplitudes considered in Figure 6 at $300 \mathrm{~km} / \mathrm{h}$ speed: these results are so close to each other that cannot be distinguished, showing that the corrugation amplitude, despite highly affecting the dynamic variation of the contact force (see Figure 6) bears almost no effect on the amplitude of the axial stresses.

In Figure 8 the vertical wheel-rail contact forces obtained for single harmonic corrugation are compared for the same vehicle speed $(300 \mathrm{~km} / \mathrm{h})$ and for different corrugation wavelengths and amplitudes: in particular, the upper subfigure shows the results obtained for a corrugation wavelength exciting the pinned-pinned resonance of the track [15], considering two corrugation amplitudes, one corresponding to the ISO 3095 limit and the other ten times greater. The peak-peak 
amplitude of the dynamic force fluctuation is approximately $81 \mathrm{kN}$, and is therefore considerably lower than for the $60 \mathrm{~mm}$ wavelength (cfr. Fig. 6), on account of the rail showing a greater mobility in this resonance condition. Also in this case, the peak-peak force amplitude is nearly proportional to the corrugation amplitude.

The lower subfigure shows the vertical contact force plotted vs. the travelled distance for a corrugation wavelength exciting the first forward bending mode of the wheelset. In this case, the peak-peak amplitude of the contact force for the larger corrugation amplitude is $82.2 \mathrm{kN}$, again significantly lower than for the $60 \mathrm{~mm}$ wavelength. In this second case however the increase of the peak-peak force amplitude with the corrugation amplitude is significantly less than proportional, and also the waveform of the contact force is different for the two corrugation amplitudes: this shall be ascribed to the different importance of the sleeper-passing effect in the two cases, rather than to the effect of non-linearities.

In Figure 9, the y-axis normal stress in the axle is shown for the same cases as in Fig. 8. For the corrugation wavelength exciting the pinned-pinned resonance of the track, no significant difference is observed with respect to the cases reported in Figure 7 for the same speed $(300 \mathrm{~km} / \mathrm{h})$, and again an increase of the corrugation amplitude leaves unaffected the amplitude of the axial stress.

However, when the corrugation wavelength exciting the wheelset resonance and the large corrugation amplitude is considered, the stress amplitude becomes $33 \%$ higher than in Figure 7 (despite the contact force being lower in this case than for the $60 \mathrm{~mm}$ wavelength) and deviates significantly form the sinusoidal waveform. This result is justified by the effect of wheelset flexibility playing a particularly important role in the case considered, on account of one mode of vibration being excited in resonance. 
A useful parameter to quantify the relevance of dynamic effects affecting the wheel-rail contact

forces and the stresses in the axle is the dynamic factor, defined as the ratio between the maximum dynamic value of the quantity considered and the corresponding static value. For the contact force, the dynamic factor $k_{Q}$ is defined according to the following equation:

$k_{Q}=\frac{Q_{d, \max }}{Q_{s}}$

with $Q_{d, \max }$ the maximum value of the contact force and $Q_{s}$ the static wheel load. For the stress in the axle the dynamic factor $k_{\sigma}$ is defined as:

$k_{\sigma}=\frac{\sigma_{d, \max }}{\sigma_{s, \max }}$

with $\sigma_{d, \max }$ maximum dynamic stress in the material point considered and $\sigma_{s, \max }$ the corresponding maximum stress under the action of rotating bending produced by the static loads acting on the wheelset.

In Figure 10 the dynamic factors $k_{Q}$ and $k_{\sigma}$ are reported as function of the vehicle speed for the different types of rail corrugation presented above, considering a corrugation amplitude ten times greater than the ISO 3095 limit; in this case, also the results for a corrugation wavelength exciting the second forward bending mode of the wheelset are presented. For the contact force dynamic factor $k_{Q}$, in the entire speed range considered, the largest values are obtained for the corrugation wavelength exciting the first forward bending mode of the wheelset, with a maximum value close to 1.8. A local maximum appears for most of the corrugation cases considered for speeds in the 125$135 \mathrm{~km} / \mathrm{h}$ range, this is due to a resonance effect associated with the sleeper passing frequency that excites P2 frequency (see P1 and P2 frequencies in Ref. [15]).- 
-As far as the stress dynamic factor $k_{\sigma}$ is concerned, very similar trends and values are obtained for the case with no roughness, for roughness wavelength exciting the pinned-pinned frequency, leading to low values of stress amplification (max. value below 1.2). Only Otherwise, in the case of the for corrugation wavelength exciting one of the wheelset the-bending resonances of the wheelset the stress dynamic factor is significantly higher and can be reaches a maximum value close up to about 1.8 for the case of corrugation wavelength exciting, with the resonance of the first forward and up to 1.4 approximately for corrugation wavelength exciting bending mode leading to larger dynamic stresses than for the second forward bending mode. The trends with speed are close to monotonically increasing, and the effect of the resonance associated with the sleeper passing is less evident than in the trend of the $k_{Q}$ dynamic factor.

These results suggest that the bending stresses in the axle are highly affected by the wavelength of rail corrugation, and that combinations of train speed and corrugation wavelength leading to a resonance of a bending mode may be especially relevant in view of axle resistance to fatigue. Particularly significant in view of real applications is the case of rail corrugation, a form of irregular wear of the rail often appearing in railway systems and characterised quasi-harmonic wear patterns developing on the rail head in longitudinal direction [18]. Corrugation wavelength can range from $\underline{50 \mathrm{~mm} \text { or less up to more than } 1 \mathrm{~m} \text { in the case of heavy haul corrugation, and typical wear depth }}$ values are in the range of some tenths of millimetre.

To quantify the relevance to fatigue of rail corrugation, we note that the design methods prescribed for railway axles by the European Standards EN13103 and EN13104 [19, 20] assume a 1.25 dynamic amplification factor on the loads generated at the primary suspension. Since the calculation of the bending stresses is then based on static equilibrium, also the bending stresses are magnified by $25 \%$ with respect to the static case, whereas the results in Figure 10 suggest that larger dynamic factors might apply when a particular combination of corrugation wavelength and train speed 
excites one bending mode. For instance, a sinusoidal rail corrugation having approximately $350 \mathrm{~mm}$ wavelength and $0.15 \mathrm{~mm}$ depth (i.e. ten times the ISO3095 limit for the considered wavelength) would produce a dynamic stress amplification close to $40 \%$. It shall be pointed out however that the equivalent static loads prescribed by EN13103/104 also include lateral wheel-rail contact forces due to curving, which are not included in the analysis presented here.

\subsection{Results for random rail corrugation and for a wheelflat}

The simple excitation cases considered in Sub-section 4.1 allow to point out the influence of different dynamic effects on train-track interaction and on the stresses in the wheelset, but do not represent realistic excitation cases. In this Sub-section, two sources of excitation typically occurring in serviced trains are considered: random rail roughness and a wheelflat. Random roughness takes place on the rail head on account of different causes: geometric imperfections associated with the manufacturing and installation of the rail, irregular wear caused by train passage and, for larger wavelengths, non-uniform track settlement due to permanent deformations in the soil and ballast (in this case, the term "track irregularities" is used instead of corrugation). Studies have shown that rail roughness and track irregularity take the form of stationary random processes characterised by their power spectral density, which can be defined based on the quality of track maintenance [2181]. On the other hand, wheelflats are localised defects occurring on the wheel surface as the result of full slip of the wheel, typically caused by poorly adjusted or faulty brakes [1922].

Figure 11 shows the vertical contact force plotted vs. the travelled distance for the train speeds of 100 and $300 \mathrm{~km} / \mathrm{h}$, considering the excitation produced by random rail corrugation (amplitude corresponding to the ISO 3095 limit). The results obtained considering a rigid and flexible wheelset model are compared. Important dynamic fluctuations of the contact force are evidenced, with the maximum dynamic value corresponding to approximately 1.6 times the static load. The differences 
between the results obtained for the rigid and flexible wheelset are relatively small in this case, and do not affect significantly the maximum contact force value.

Figure 12 presents the vertical contact force vs. travelled distance for a vehicle affected by a wheelflat. The calculation has been carried out for 50 and $300 \mathrm{~km} / \mathrm{h}$ by means of the rigid and enhanced wheelset models. A severe dynamic effect is observed, consisting of the complete loss of contact between the wheel and the rail, followed by an impact leading to a maximum value of the contact force which is between 3 and 4 times the static load and finally by a transient vibration. The differences between the results for the rigid and flexible wheelset model are small in terms of duration of the contact loss and of maximum overloading, but the transient following the impact is affected quite remarkably by wheelset flexibility, as demonstrated by the different frequency contents of the contact force signal, see particularly the zoomed view on the right side of the figure.

The y-axis stresses for the random rail corrugation and wheelflat excitation cases are shown in Figure 13. In the random corrugation case, the dynamic fluctuations of the stress component are relatively low and lead to a maximum peak-peak stress amplitude around 91.2 MPa, corresponding to a dynamic amplification with respect to the maximum stress produced by the static load $k_{\sigma}$ of 1.18 approximately. When the wheelflat defect is considered, the results are highly affected by the vehicle speed, with large dynamic effects taking place at low speed (with a dynamic factor $k_{\sigma}$ of 1.34 approximately) on account of the repeated loss of contact of the wheel to the rail, and with a reduction of the dynamic stresses at higher speed.

Finally, in Figure 14 the dynamic factors $k_{Q}$ and $k_{\sigma}$ are reported for the random corrugation and wheelflat as function of the vehicle speed. The contact force dynamic factor $k_{Q}$ is nearly monotonically increasing up to a maximum value close to 1.8 for random corrugation excitation, whereas in case of wheelflat excitation the dynamic factor is much higher, in the range of 2.8-4.2, 
with larger values occurring at lower speeds: this is because at low speed the duration of the contact loss caused by the wheelflat is longer and hence the following impact is larger. The stress dynamic factor $k_{\sigma}$ for the random corrugation excitation case is almost monotonically increasing with speed, with a maximum value close to 1.3 . For the wheelflat excitation case, a completely different trend is observed, the stress dynamic factor $k_{\sigma}$ being initially decreasing with the speed and then increasing above $200 \mathrm{~km} / \mathrm{h}$. The maximum value of the stress dynamic factor for this excitation case is obtained at the lowest speed considered in the analysis and is slightly below 1.5.

As in the case of excitation generated by a single-harmonic rail corrugation, it is interesting to observe that in some cases the dynamic factor $k_{\sigma}$ exceeds the 1.25 value assumed in EN13103/104. For wheelflat excitation, this happens at low speeds (below $75 \mathrm{~km} / \mathrm{h}$ ) which are typical e.g. of freight application whereas for random rail roughness the 1.25 value is exceeded only at very high speeds above $300 \mathrm{~km} / \mathrm{h}$, which are only relevant to very high speed trains. It shall be stressed however that the results shown in Figure 14 depend on the amplitude of the defects being considered and, in case of more severe irregularities, larger dynamic stresses shall be expected.

It is also interesting to point out that by using a static calculation to derive the bending stresses, Standards EN13103/104 inherently imply a proportionality between the contact forces and the $\underline{\text { stresses, whereas the results in figures } 10 \text { and } 14 \text { show that the stress dynamic factor }} k_{\sigma} \underline{\text { is }}$ generally lower (sometimes much lower) than the dynamic factor for the vertical contact force $k_{Q}$. This circumstance is due to the inertia forces generated in the wheels and to the different magnification of wheelset flexible modes produced when resonance conditions occur and suggests that a method based on a static calculation could be not fully suited to estimate service stresses in $\underline{\text { the axle. Dynamic train-track interaction models such as the one proposed in this paper could be }}$ envisaged as a means to derive a more realistic estimate of service stresses in the axle, but this 
requires the study to be extended to consider the effect of curving, which is envisaged as a next development of the work described here.

\section{CONCLUSIONS}

Dynamic effects may be extremely important in determining the fatigue resistance of railway axles, and need to be properly considered in the axle design process. This paper has presented a method for the numerical estimation of the dynamic stresses in railway axles, based on the simulation of the interaction between a flexible wheelset and a flexible track. The method is presently limited to the case of tangent track running of the wheelset, but the extension to the curving condition is envisaged as a next step of the research, to consider the additional axle loading due to wheel-rail contact forces in a curve.

Results of the numerical procedures were presented in the paper, considering the dynamic excitation caused by rail corrugation and by a wheelflat for different wheelset speed values. The results clearly show that dynamic effects may lead to a significant dynamic amplification of the stresses in the axle, which is in some cases close to $70 \%$. However, the actual relevance of dynamic effects affecting axle stresses is strongly depending upon the type of excitation and the vehicle speed.

When single harmonic corrugation is considered, the dynamic amplification of axle stresses is relatively low except in the case when the corrugation wavelength excites the bending modes of the wheelset. In the case of random multi-harmonic rail corrugation, the dynamic amplification factors are relatively low because the excitation is spread over a wide range of frequencies, thus reducing the importance of resonance effects. In all rail corrugation cases considered, regardless the waveform of the excitation, the dynamic stress amplification tends to increase with the vehicle speed, so that the most critical case is obtained for high-speed applications. 


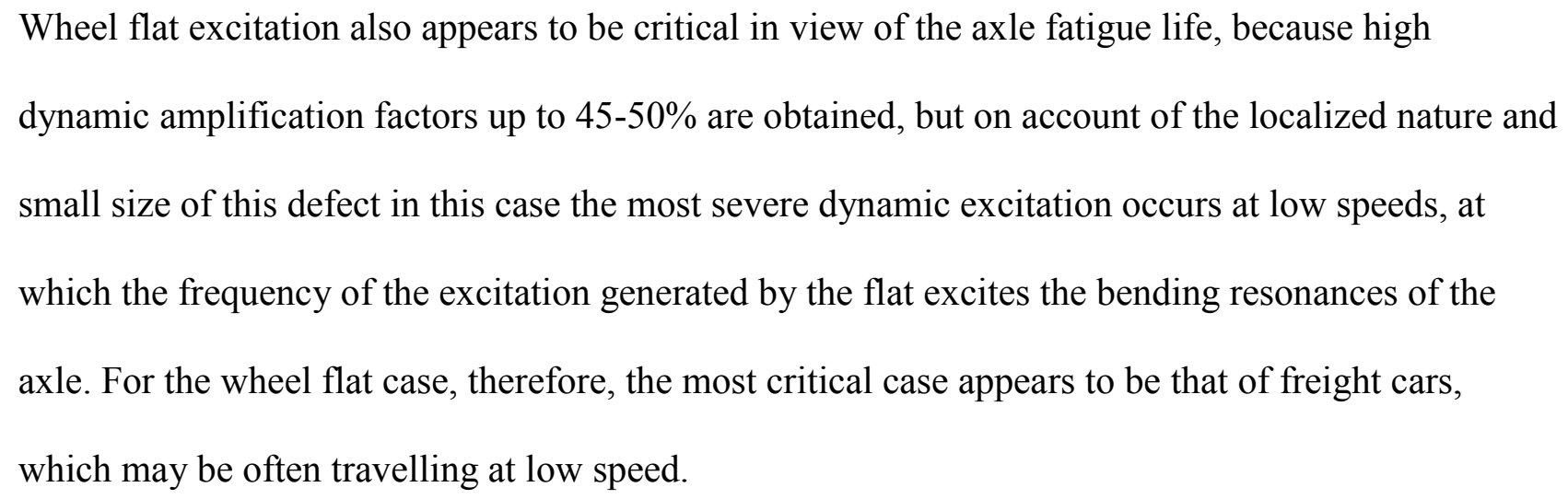

Overall, the analyses presented in this paper show that dynamic effects are essential to correctly estimate the wheelset's fatigue life. More research and validation is needed to transfer the findings presented here into axle design practices. Hopefully however the paper has highlighted an important area for future research, in view of further improving the reliability of railway transport.

\section{Acknowledgements}

The authors gratefully acknowledge the support for this work provided by the Project TRA201015669 (Ministerio de Ciencia e Innovación) and TRA2007-67167 (Ministerio de Educación y Ciencia-FEDER). 


\section{References}

[1] R. A. Smith, Railway fatigue failures: an overview of a long standing problem, Materialwissenschaft und Werkstofftechnik 36:11 (2005) 697-705

[2] V. Grubisic, G. Fischer, Procedure for reliable durability validation of train axles, Materialwissenschaft und Werkstofftechnik 37:12 (2006) 973-982

[3] S. Beretta, M. Carboni Variable amplitude fatigue crack growth in a mild steel for railway axles: Experiments and predictive models, Engineering Fracture Mechanics 78 (2011) 848862.

[4] M. Luke, I. Varfolomeev, K. Lüthepol, A. Esderts, Fatigue crack growth in railway axles: Assessment concept and validation tests, Engineering Fracture Mechanics 78 (2011) 714730.

[5] V. Grubisic, G. Fischer, Bemessung von Radsatzwellen - Einflussgrssen und Vorgehen bei der Auslegung. Bericht FB-226, LBF, Darmstadt; 2005.

[6] S. Alfi, F. Braghin, S. Bruni, Numerical and experimental evaluation of extreme loads for improved wheelset design, Vehicle System Dynamics, 46 S (2008) 431-444.

[7] A. S. Watson, K. Timmis, A method of estimating railway axle stress spectra, Engineering Fracture Mechanics 78 (2011) 836-847.

[8] S. Bruni, R. Corradi, L. Mazzola, Wheel rail contact forces as input for optimal and robust axle design, 8th International Conference on Contact Mechanics and Wear of Rail/Wheel Systems (CM2009), Firenze, Italy, September 15-18, 2009.

[9] D.J. Thompson, C.J.C. Jones Thompson A review of the modelling of wheel/rail noise generation, Journal of Sound and Vibration, 231 (2000), pp. 519-536.

[10] J.C.O. Nielsen, A. Igeland, Vertical dynamic interaction between train and track-influence of wheel and track imperfections, Journal of Sound and Vibration 187 (5) (1995) 825-839.

[11] G. Diana, F. Cheli, S. Bruni, A. Collina, Experimental and numerical investigation on subway short pitch corrugation, Vehicle System Dynamics, 29 S, (1998) 234-245. 
[12] J.C.O. Nielsen, J. Oscarsson, Simulation of dynamic train_track interaction with statedependent track properties, Journal of Sound and Vibration 275 (2004) 515-532.

[13] J. Fayos, L. Baeza, J. E. Tarancón and F. D. Denia, An Eulerian coordinate-based method for analysing the structural vibrations of a solid of revolution rotating about its main axis, Journal of Sound and Vibration 306 (2007) 618-635

[14] L. Baeza, J. Fayos, A. Roda and R. Insa, High frequency railway vehicle-track dynamics through flexible rotating wheelsets, Vehicle System Dynamics 46 (2008) 647 - 659

[15] Baeza, L., Huajiang, O., A railway track dynamics model based on modal substructuring and cyclic boudary condition, Journl of Sound and V-ibration 330 (2011) 75-86.

[16] L. Baeza, A. Roda, J.C.O. Nielsen, Railway vehicle/track interaction analysis using a modal substructuring approach, Journal of Sound and Vibration 293 (2006) 112-124

[17] J.J. Kalker, A fast algorithm for the simplified theory of rolling contact, Vehicle System Dynamics 11 (1982) 1-13.

[18] S. L. Grassie, J. Kalousek, Rail corrugation: characteristics, causes and treatments, Proc. Instn. Mech. Engrs. Part F - Journal of Rail and Rapid Transit, 207 (1993), 57-68.

[19] EN 13103 Railway applications - Wheelsets and bogies - Non-powered axles - Design method, CEN, Brussels, April 2001.

[20] EN 13104 Railway applications - Wheelsets and bogies - Powered axles - Design method, CEN, Brussels, April 2001.

[1821] ORE B 176: Bogies with steered or steering wheelsets. Report No. 1: Specifications and preliminary studies, Vol. 2, Specification for a bogie with improved curving characteristics. ORE, Utrecht 1989.

[2219] A. Johansson, J.C.O. Nielsen, Out-of-round railway wheels - wheel-rail contact forces and track response derived from field tests and numerical simulations, Proc. Instn. Mech. Engrs. 
Part F - Journal of Rail and Rapid TransitProc. IMechE Part F: J. Rail and Rapid Transit, $217 \underline{(2003)}, 135-146,2003$. 
List of figure captions

Figure 1: The train-track interaction model.

Figure 2: The cyclic track model.

Figure 3: Detail of the track model. Left: model of some sleeper bays. Right:sleeper and railpad model.

Figure 4: Finite element model of the flexible wheelset.

Figure 5: Studied section of wheelset and spatial point where the stresses have been calculated.

Figure 6: Wheel-rail contact force when the vehicle circulates on a corrugated track with corrugation wavelength $60 \mathrm{~mm}$. Above: effect of speed and of wheelset flexibility for corrugation amplitude corresponding to the ISO 3095 limit. Below: effect of corrugation amplitude for speed $300 \mathrm{~km} / \mathrm{h}$ and flexible wheelset.

Figure 7: y-axis normal stress in the studied section of the axle (cfr. Fig. 5) when the vehicle circulates on a corrugated track with corrugation wavelength $60 \mathrm{~mm}$. Left: two vehicle speeds, corrugation amplitude corresponding to the ISO 3095 limit. Right: two corrugation amplitudes (the results are undistinguishable).

Figure 8: Wheel-rail contact force when the vehicle circulates on a corrugated track with different corrugation wavelengths. Two corrugation amplitudes are considered, corresponding to the ISO 3095 limit and 10 times the ISO 3095 limit. Above: the corrugation wavelength excites the pinned-pinned mode of the rail. Below: the corrugation wavelength excites the first forward bending mode of the wheelset.

Figure 9: y-axis normal stress in the studied section of the axle (cfr. Fig. 5) when the vehicle circulates on a corrugated track at $300 \mathrm{~km} / \mathrm{h}$ speed. Left: the corrugation wavelength excites the pinned-pinned mode of the rail. Right: the corrugation wavelength excites the first forward bending mode of the wheelset.

Figure 10: Dynamic factors $k_{Q}$ (vertical contact force, upper subfigure) and $k_{\sigma}$ (y-axis stress, lower subfigure) for different excitation cases and vehicle velocities. The roughness amplitude is ten times higher the ISO 3095 limit.

Figure 11: Wheel-rail contact force when the vehicle circulates at 100 and $300 \mathrm{~km} / \mathrm{h}$ speeds on a randomly corrugated track.

Figure 12: Wheel-rail contact force when the vehicle circulates at 50 (above) and $300 \mathrm{~km} / \mathrm{h}$ (below) speeds on a perfectly even rail in presence of a $50 \mathrm{~mm}$ wheelflat.

Figure 13: y-axis normal stress in the studied section of the axle (cfr. Fig. 5) when the vehicle circulates at $300 \mathrm{~km} / \mathrm{h}$ speed on a randomly corrugated track (left) and on a perfectly even rail in presence of a $50 \mathrm{~mm}$ wheelflat at $50 \mathrm{~km} / \mathrm{h}$ (centre) and $300 \mathrm{~km} / \mathrm{h}$ (right).

Figure 14: Dynamic factors $k_{Q}$ (vertical contact force, upper subfigure) and $k_{\sigma}$ (y-axis stress, lower subfigure) for the random corrugation and wheelflat excitation cases. 
Table captions

1

2

3

4

5

6

7

8

9

10

11

12

13

14

15

16

17

18

19

20

21

22

23

24

25

26

27

28

29

30

31

32

33

34

35

36

37

38

39

40

41

42

43

44

45

46

47

48

49

50

51

52

53

54

55

56

57

58

59

60

61

62

63

64

65

Table 1: Simulation parameters. 


\begin{tabular}{|l|l|l|l|}
\hline \multicolumn{2}{|c|}{ Wheelset model data } & \multicolumn{2}{c|}{ Track model data } \\
\hline Mass of wheelset & $1375 \mathrm{~kg}$ & Sleeper bay & $0.6 \mathrm{~m}$ \\
\hline Axle load & $120 \mathrm{kN}$ & Sleeper number & 70 \\
\hline $\begin{array}{l}\text { Primary suspension longitudinal } \\
\text { stiffness }\end{array}$ & $7.5 \mathrm{MN} / \mathrm{m}$ & Sleeper mass & $324 \mathrm{~kg}$ \\
\hline Primary suspension lateral stiffness & $7.1 \mathrm{MN} / \mathrm{m}$ & Track bed stiffness & $200 \mathrm{MN} / \mathrm{m}$ \\
\hline $\begin{array}{l}\text { Primary suspension vertical } \\
\text { stiffness }\end{array}$ & $0.81 \mathrm{MN} / \mathrm{m}$ & Track bed damping & $150 \mathrm{kNs} / \mathrm{m}$ \\
\hline $\begin{array}{l}\text { Primary suspension vertical } \\
\text { damping }\end{array}$ & $30 \mathrm{kNs} / \mathrm{m}$ & Rail pad stiffness & $1 \mathrm{GN} / \mathrm{m}$ \\
\hline $\begin{array}{l}\text { Primary suspension longitudinal } \\
\text { damping }\end{array}$ & $100 \mathrm{kNs} / \mathrm{m}$ & Rail pad damping & $50 \mathrm{kNs} / \mathrm{m}$ \\
\hline $\begin{array}{l}\text { Primary suspension lateral } \\
\text { damping }\end{array}$ & $100 \mathrm{kNs} / \mathrm{m}$ & Rail section & $\mathrm{UIC60}$ \\
\hline
\end{tabular}

Table 1: Simulation parameters. 


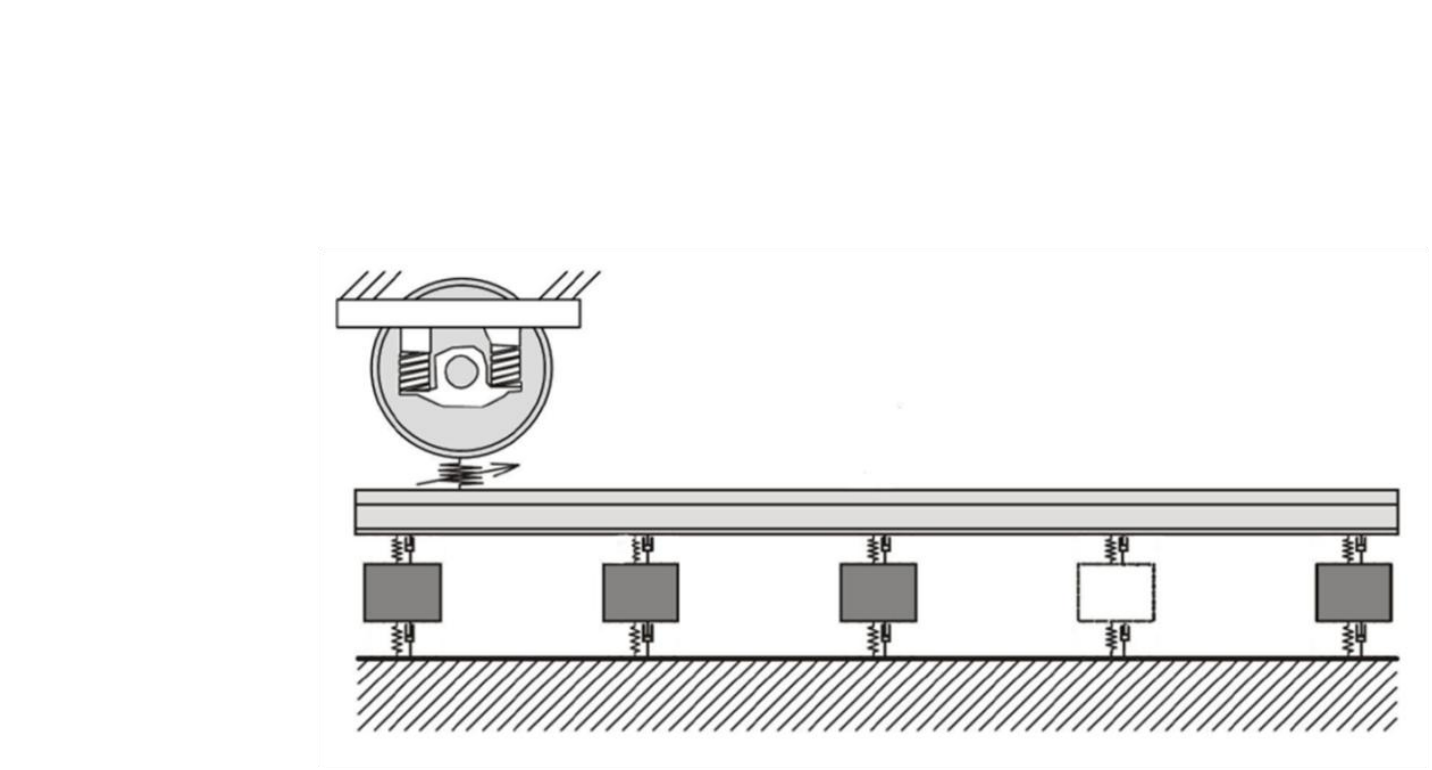

Figure 1: The train-track interaction model.

Figure 1

Figure

.

$\sqrt{2}$

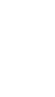

$\sqrt{2}$

$\sqrt{2}$

$\sqrt{2}$

.
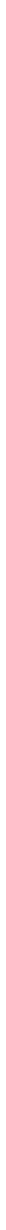


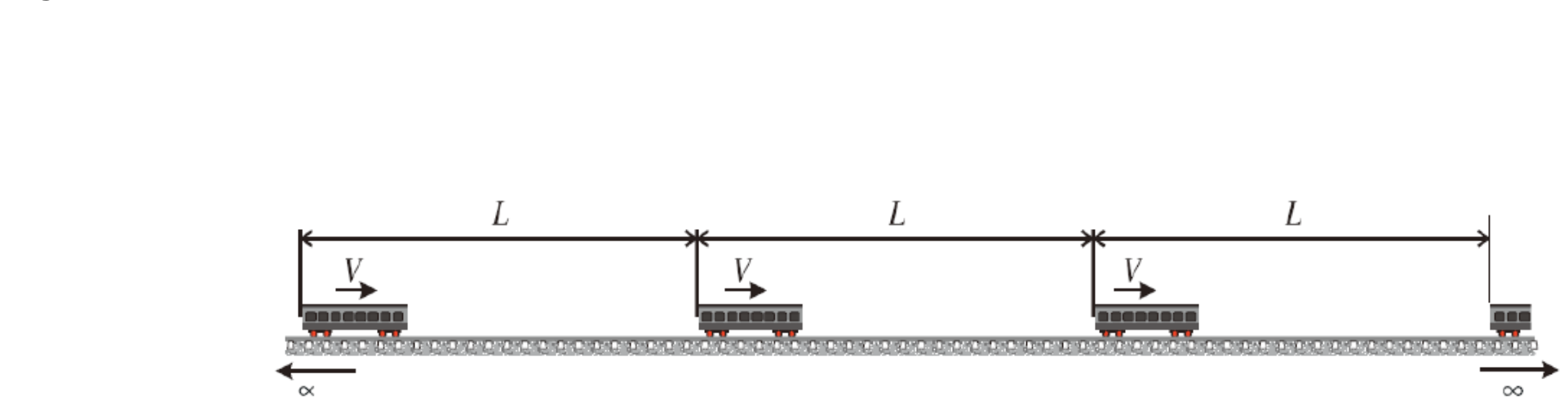

Figure 2: The cyclic track model.

Figure 2

Figure 2: The cyclic track model.

$\infty$

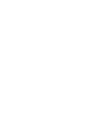

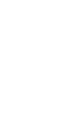
. 
Figure 3
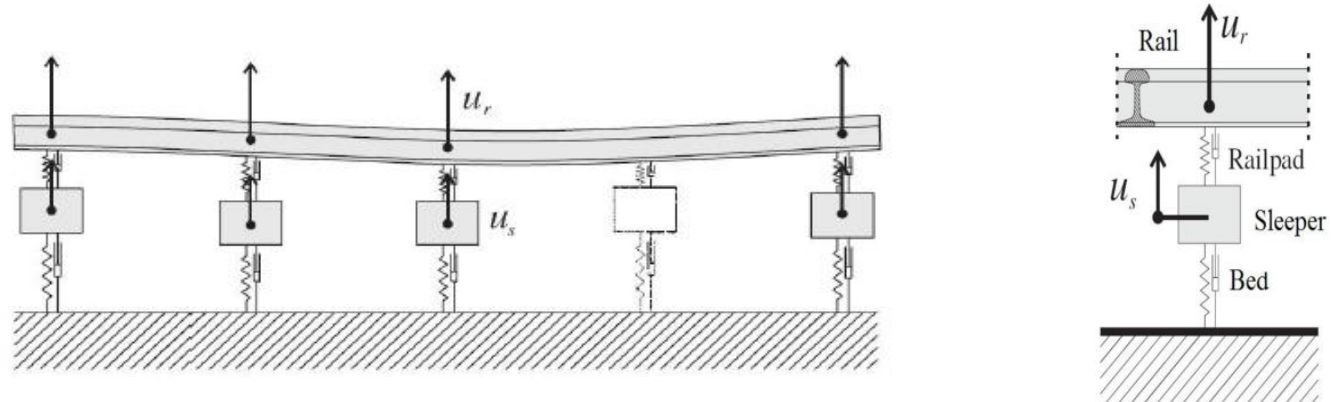

Figure 3: Detail of the track model. Left: model of some sleeper bays. Right:sleeper and railpad model. 
Figure 4

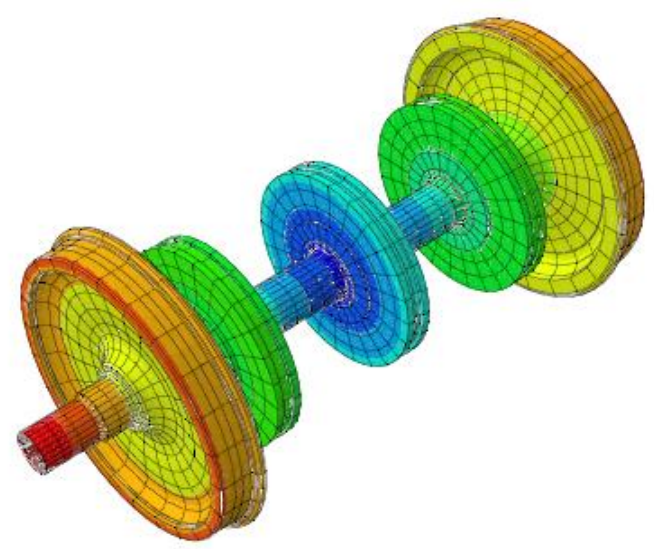

Figure 4: Finite element model of the flexible wheelset. 
Figure 5
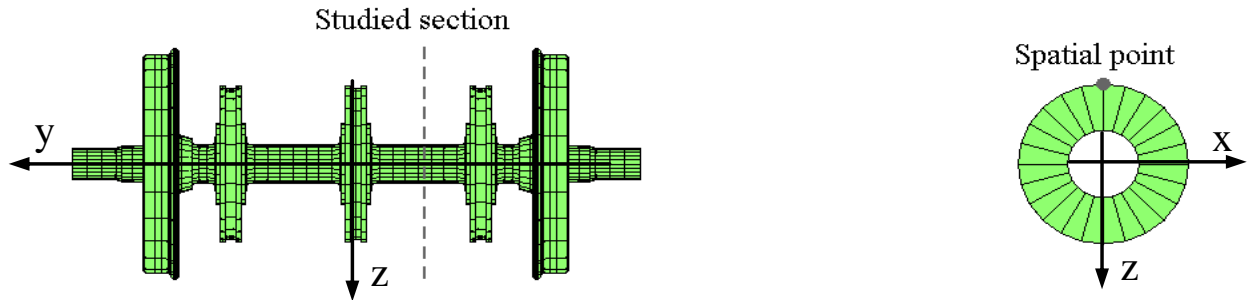

Figure 5: Studied section of wheelset and spatial point where the stresses have been calculated. 


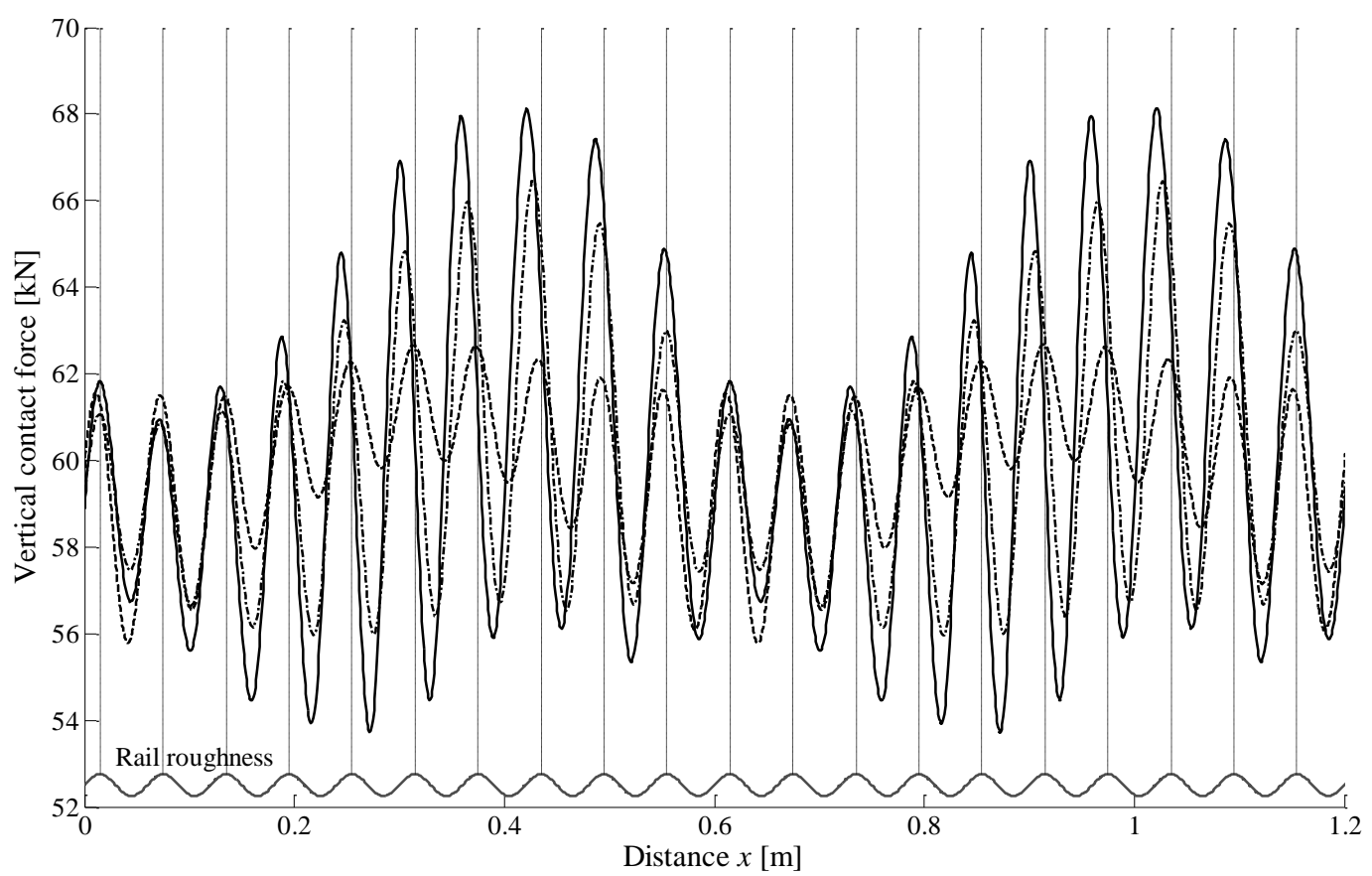

Flexible wheelset $V=100 \mathrm{~km} / \mathrm{h} \quad-\cdots \cdots \cdot$ Flexible wheelset $V=300 \mathrm{~km} / \mathrm{h}$

Rigid wheelset $V=300 \mathrm{~km} / \mathrm{h}$

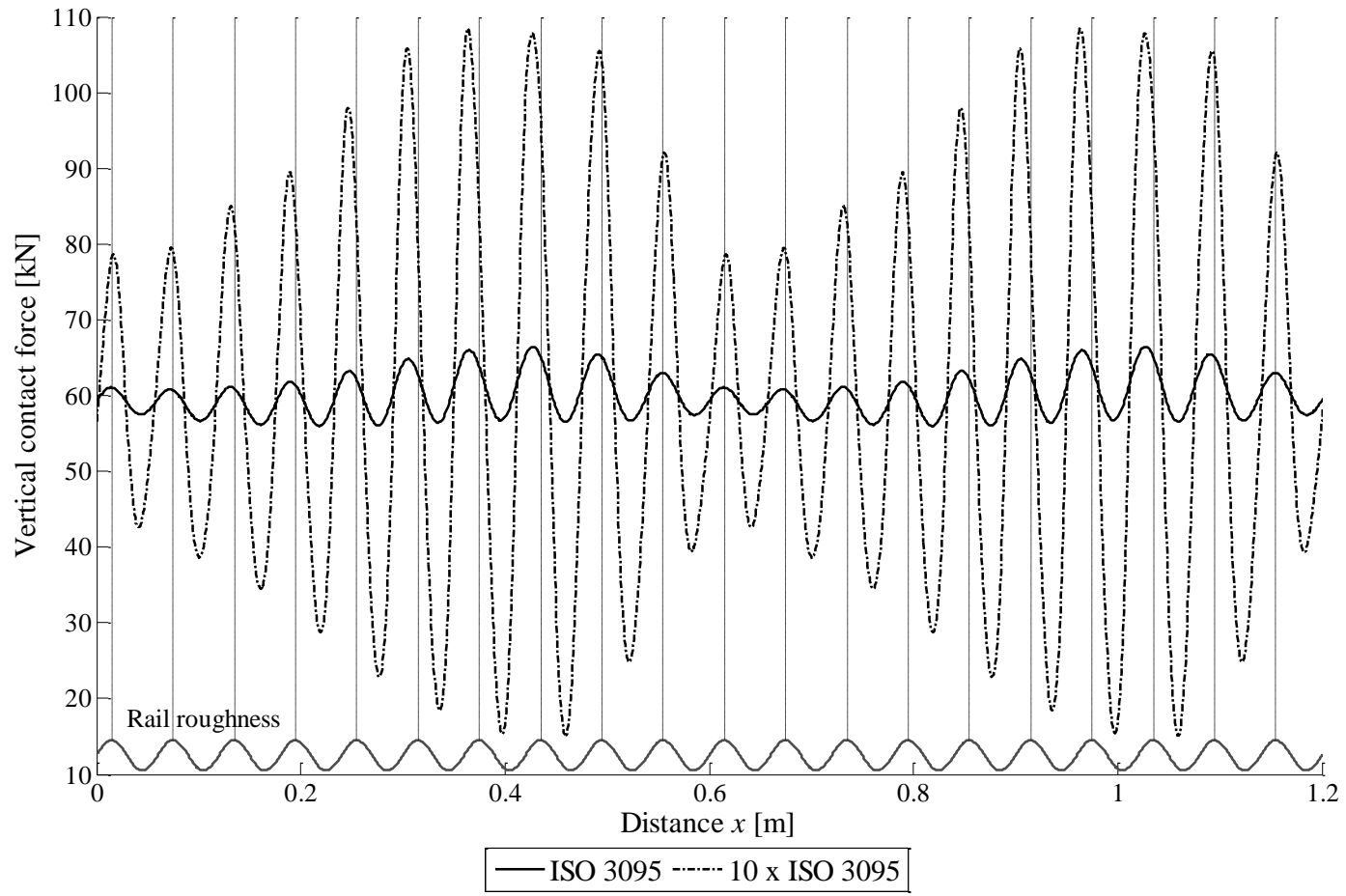

Figure 6: Wheel-rail contact force when the vehicle circulates on a corrugated track with corrugation wavelength $60 \mathrm{~mm}$. Above: effect of speed and of wheelset flexibility for corrugation amplitude corresponding to the ISO 3095 limit. Below: effect of corrugation amplitude for speed $300 \mathrm{~km} / \mathrm{h}$ and flexible wheelset. 

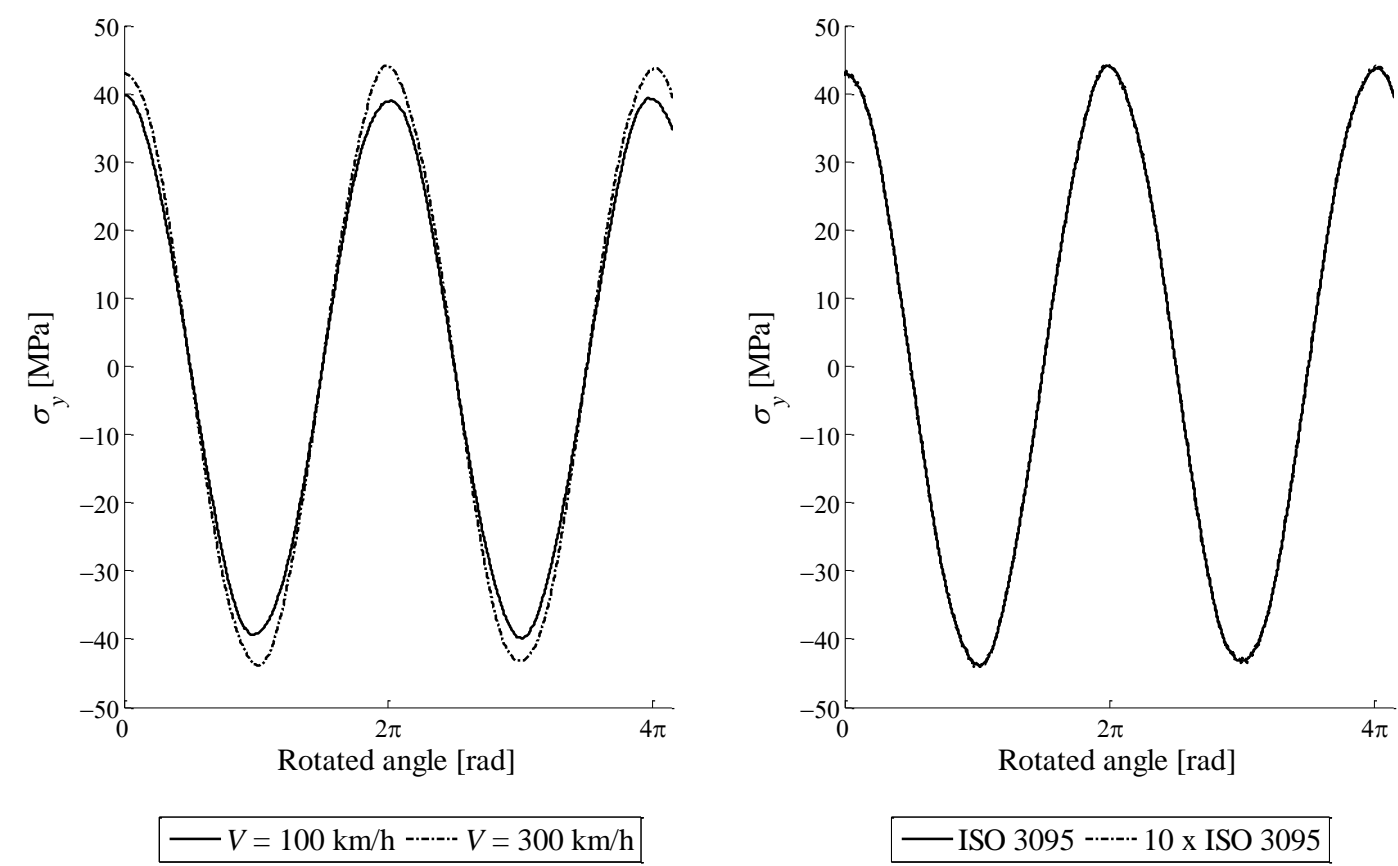

Figure 7: y-axis normal stress in the studied section of the axle (cfr. Fig. 5) when the vehicle circulates on a corrugated track with corrugation wavelength $60 \mathrm{~mm}$. Left: two vehicle speeds, corrugation amplitude corresponding to the ISO 3095 limit. Right: two corrugation amplitudes (the results are undistinguishable). 

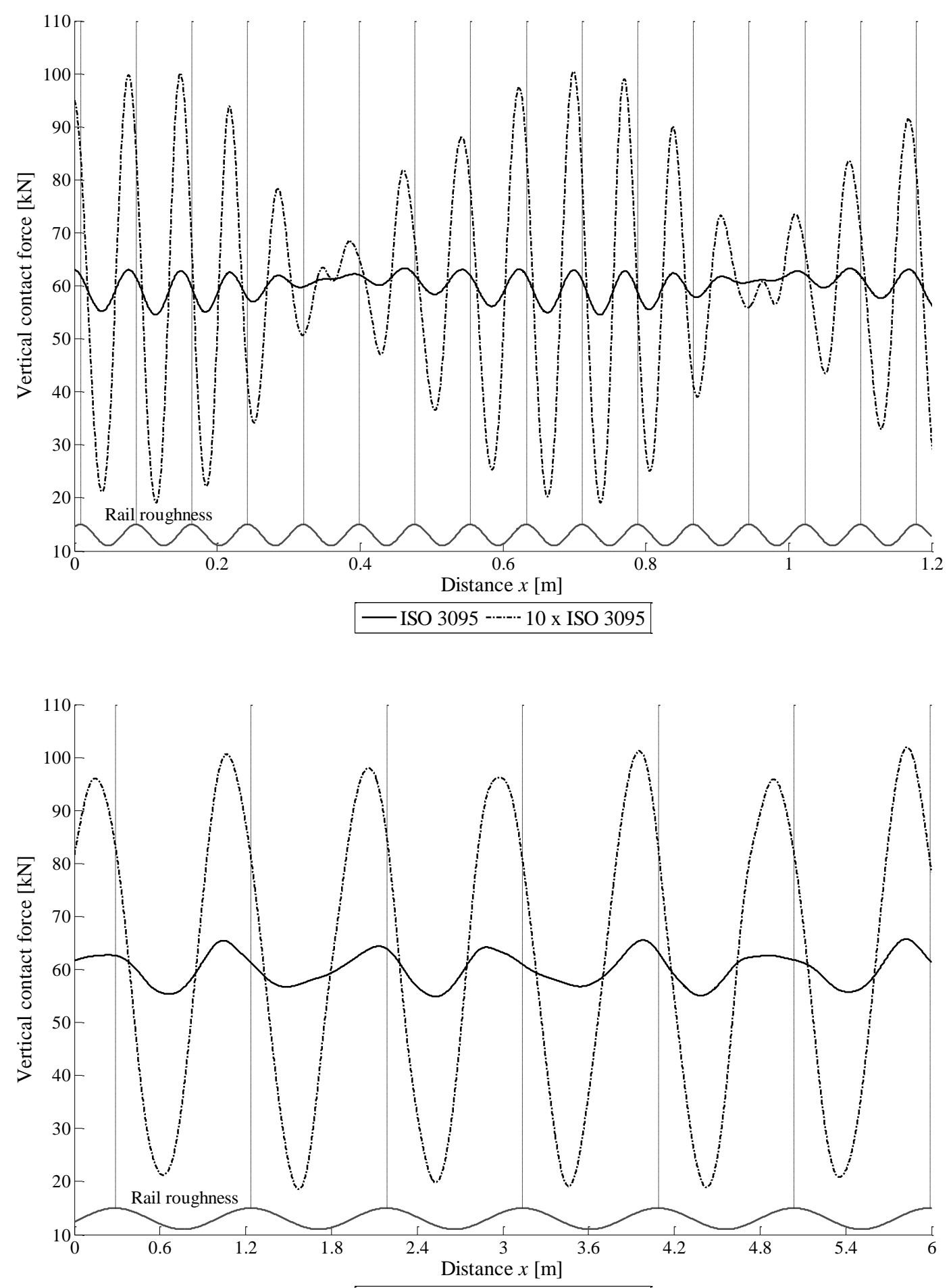

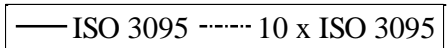

Figure 8: Wheel-rail contact force when the vehicle circulates on a corrugated track with different corrugation wavelengths. Two corrugation amplitudes are considered, corresponding to the ISO 3095 limit and 10 times the ISO 3095 limit. Above: the corrugation wavelength excites the pinned-pinned mode of the rail. Below: the corrugation wavelength excites the first forward bending mode of the wheelset. 


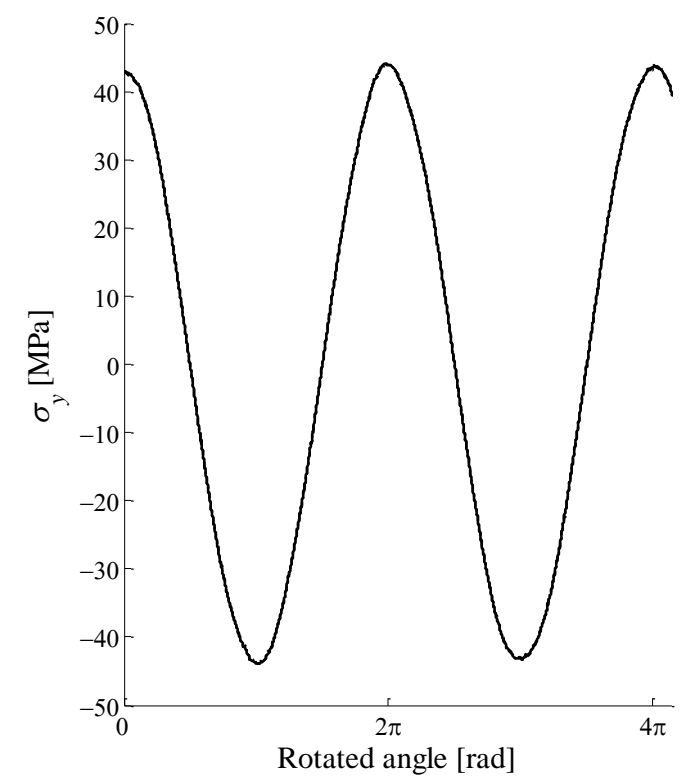

- ISO $3095 \quad \cdots-\cdots \cdot 10$ x ISO 3095

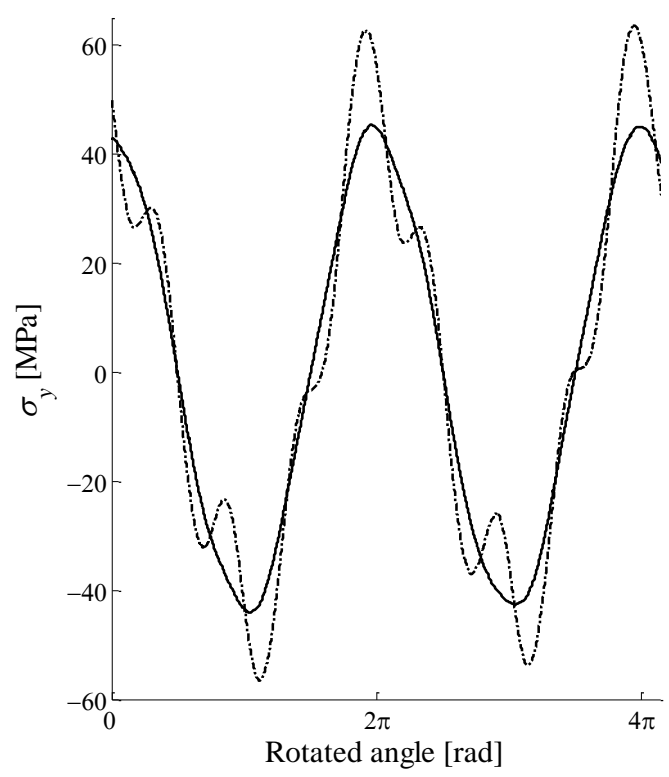

- ISO 3095 -..... 10 x ISO 3095

Figure 9: y-axis normal stress in the studied section of the axle (cfr. Fig. 5) when the vehicle circulates on a corrugated track at $300 \mathrm{~km} / \mathrm{h}$ speed. Left: the corrugation wavelength excites the pinned-pinned mode of the rail. Right: the corrugation wavelength excites the first forward bending mode of the wheelset. 

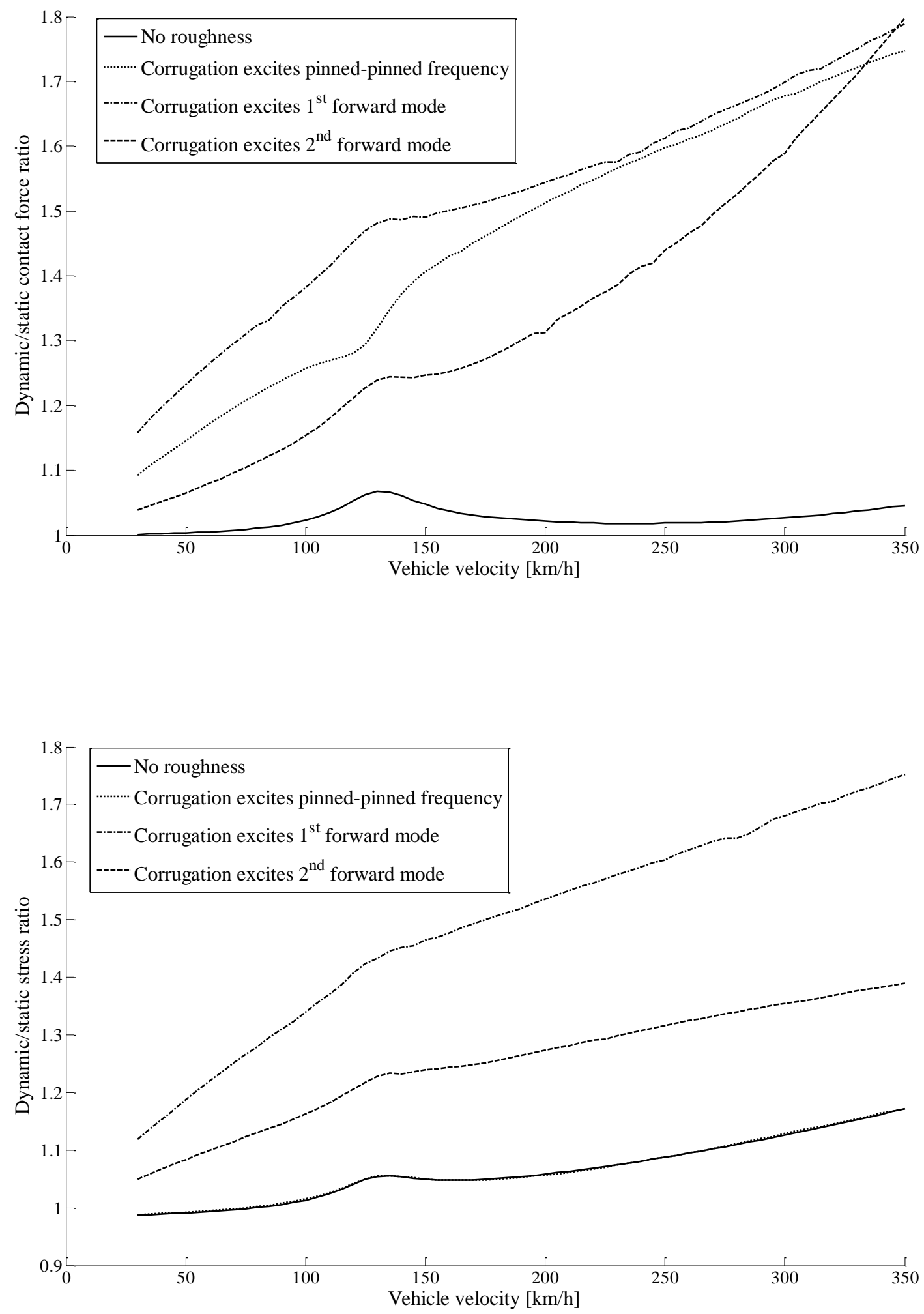

Figure 10: Dynamic factors $k_{Q}$ (vertical contact force, upper subfigure) and $k_{\sigma}$ (y-axis stress, lower subfigure) for different excitation cases and vehicle velocities. The roughness amplitude is ten times higher the ISO 3095 limit. 


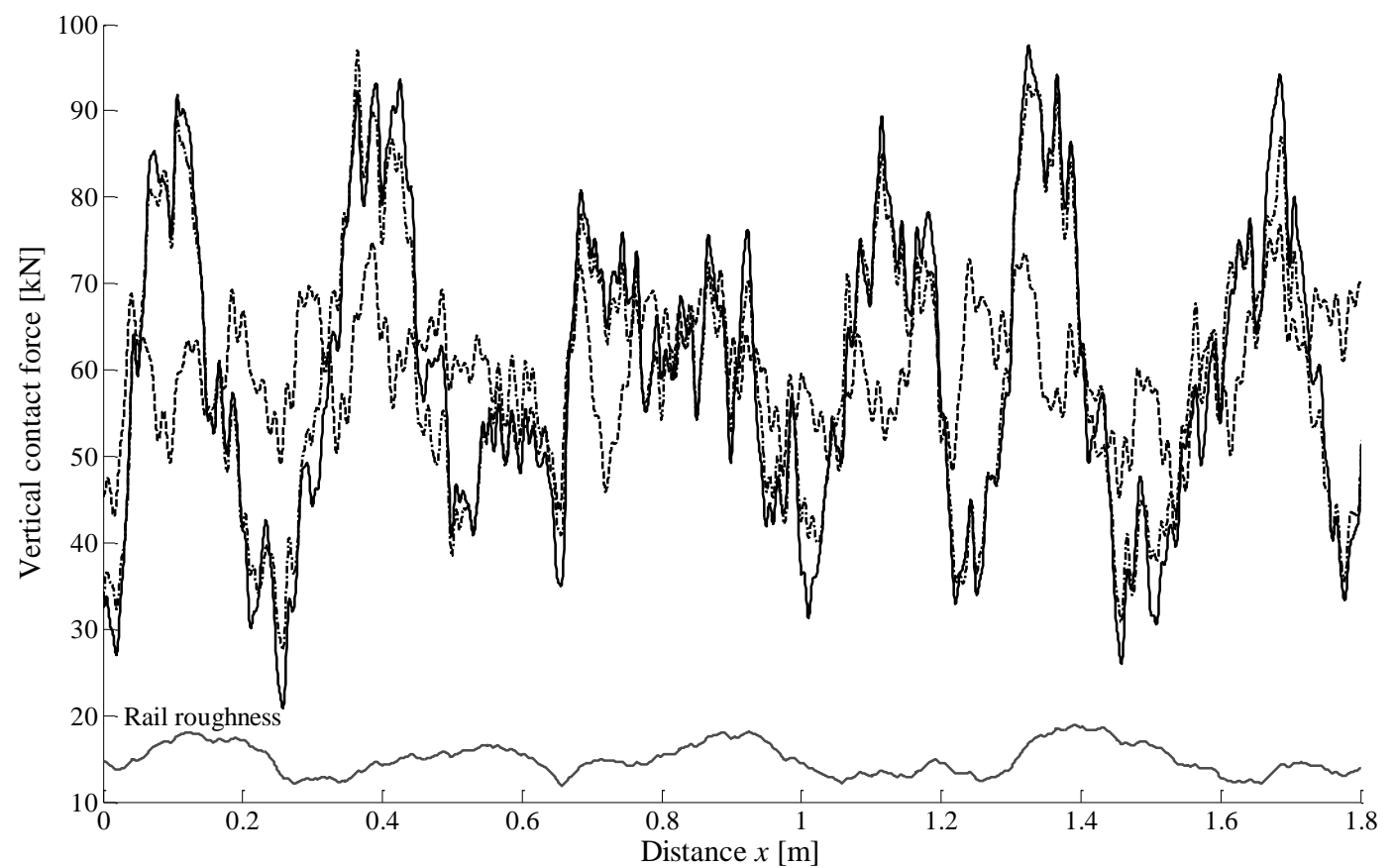

------ Flexible wheelset $V=100 \mathrm{~km} / \mathrm{h}$

Flexible wheelset $V=300 \mathrm{~km} / \mathrm{h}$

Rigid wheelset $V=300 \mathrm{~km} / \mathrm{h}$

Figure 11: Wheel-rail contact force when the vehicle circulates at 100 and $300 \mathrm{~km} / \mathrm{h}$ speeds on a randomly corrugated track. 

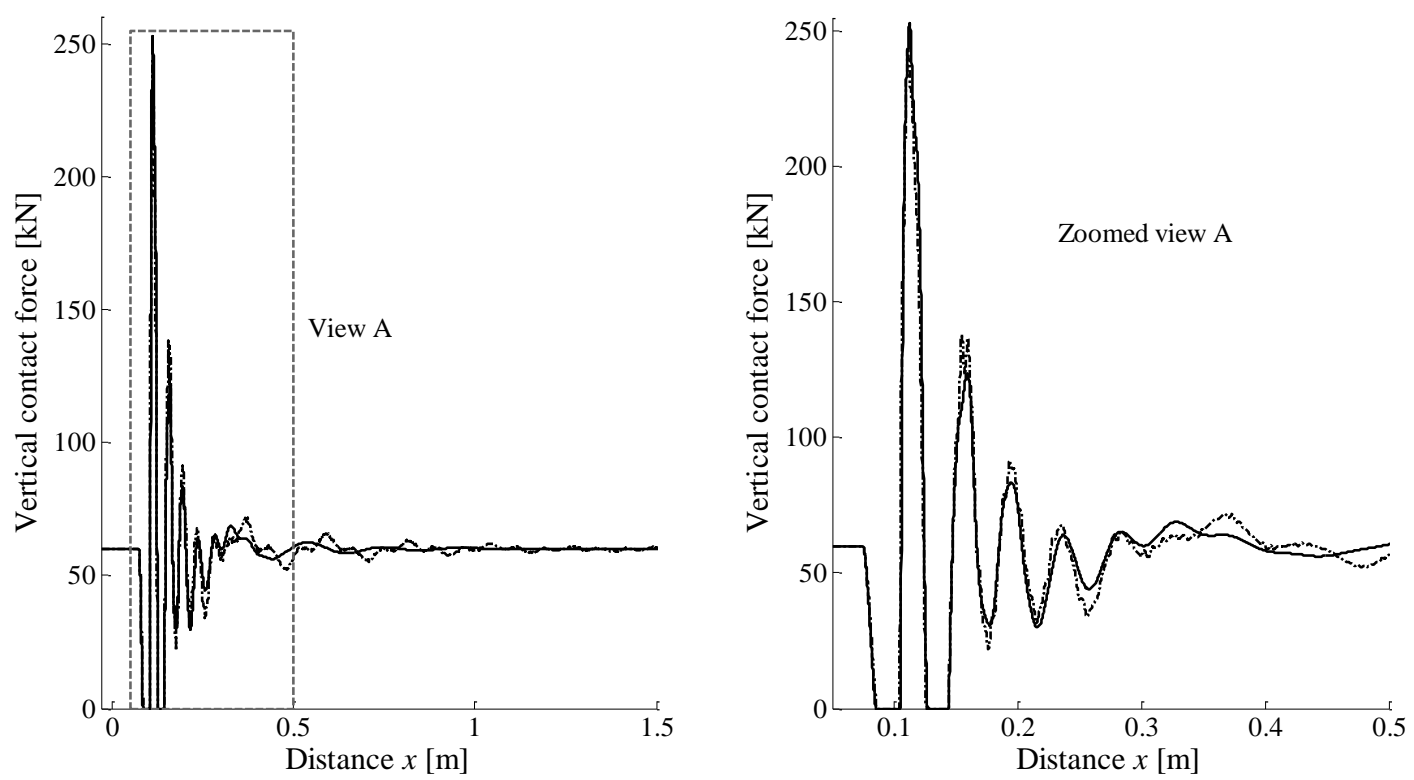

----- Flexible wheelset $V=50 \mathrm{~km} / \mathrm{h}$ - Rigid wheelset $V=50 \mathrm{~km} / \mathrm{h}$
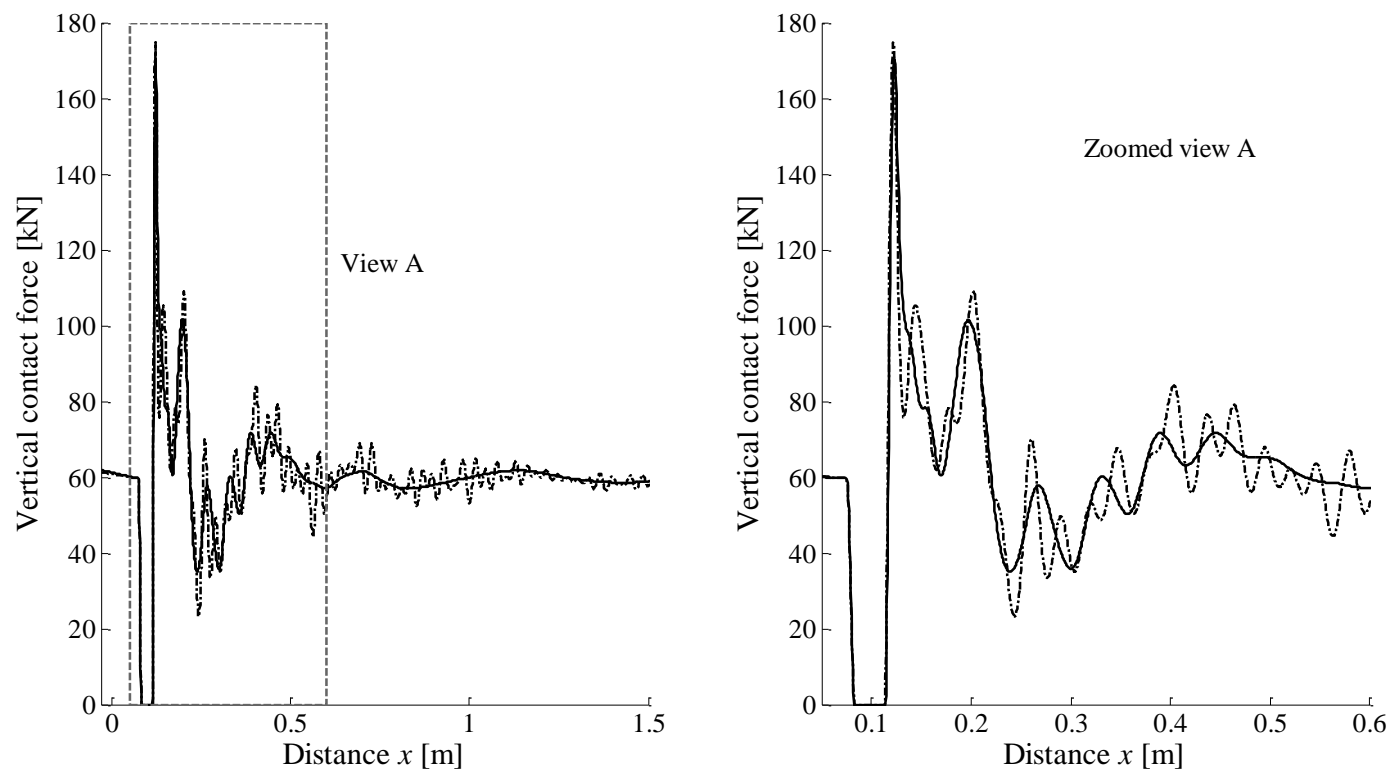

---- Flexible wheelset $V=300 \mathrm{~km} / \mathrm{h}$ - Rigid wheelset $V=300 \mathrm{~km} / \mathrm{h}$

Figure 12: Wheel-rail contact force when the vehicle circulates at 50 (above) and 300 $\mathrm{km} / \mathrm{h}$ (below) speeds on a perfectly even rail in presence of a $50 \mathrm{~mm}$ wheelflat. 

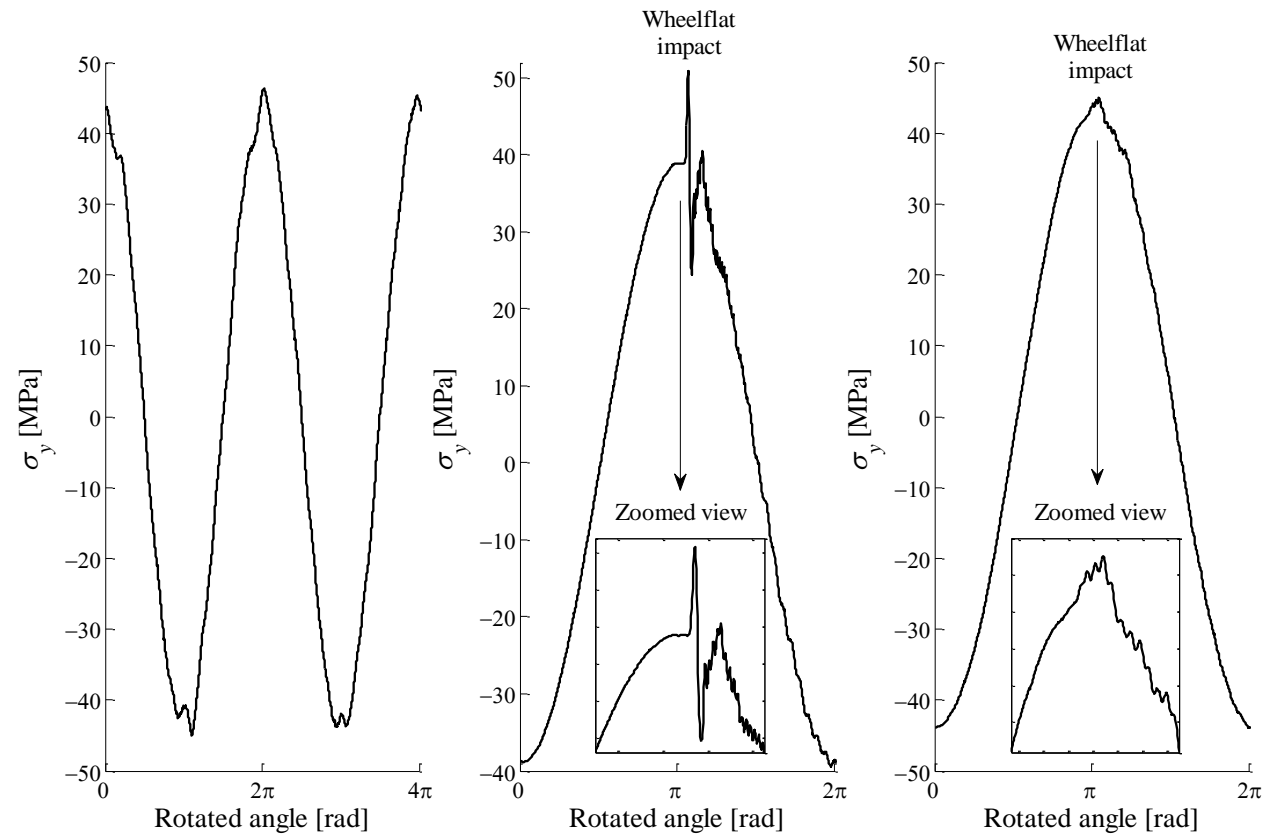

Figure 13: y-axis normal stress in the studied section of the axle (cfr. Fig. 5) when the vehicle circulates at $300 \mathrm{~km} / \mathrm{h}$ speed on a randomly corrugated track (left) and on a perfectly even rail in presence of a $50 \mathrm{~mm}$ wheelflat at $50 \mathrm{~km} / \mathrm{h}$ (centre) and $300 \mathrm{~km} / \mathrm{h}$ (right). 

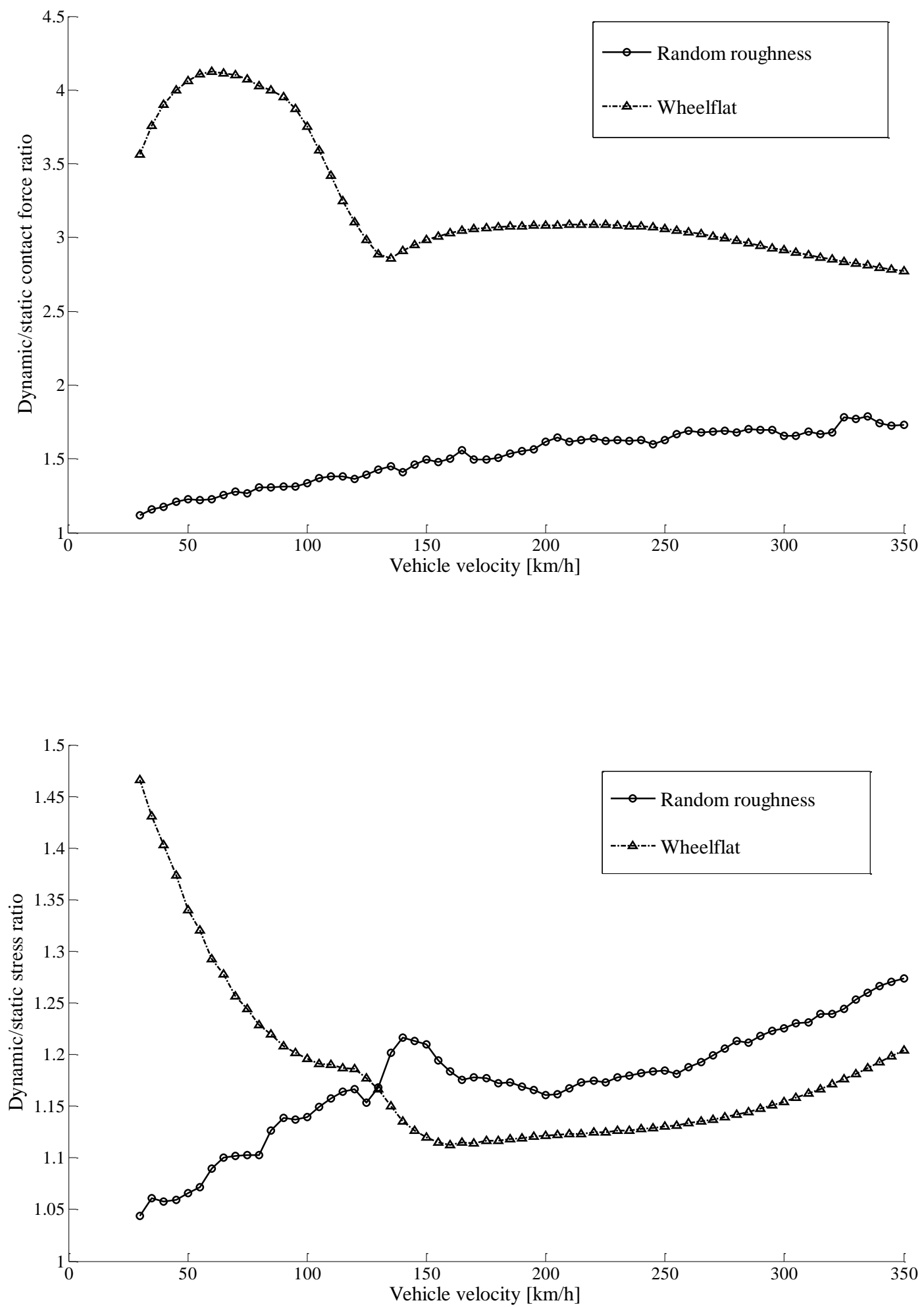

Figure 14: Dynamic factors $k_{Q}$ (vertical contact force, upper subfigure) and $k_{\sigma}$ (y-axis stress, lower subfigure) for the random corrugation and wheelflat excitation cases. 